\title{
Testing the inflationary slow-roll condition with tensor modes
}

\author{
William Giarè, ${ }^{1, *}$ Eleonora Di Valentino, ${ }^{2, \dagger}$ and Alessandro Melchiorri ${ }^{1, \$}$ \\ ${ }^{1}$ Physics Department and INFN, Università di Roma "La Sapienza," Ple Aldo Moro 2, 00185, Rome, Italy \\ ${ }^{2}$ Jodrell Bank Center for Astrophysics, School of Physics and Astronomy, University of Manchester, \\ Oxford Road, Manchester, M13 9PL, United Kingdom
}

(Received 14 February 2019; published 19 June 2019)

\begin{abstract}
A major goal of modern cosmology is the detection of $B$-modes in the cosmic microwave background polarization originated from primordial gravitational waves. Their detection not only could provide substantial evidence for primordial inflation but also could shed light on its physical nature. Under the assumption of single-field slow-roll inflation, a set of conditions exist for the scalar and tensor parameters. In particular, given a constraint on the scalar spectral index $n_{\mathrm{s}}$, its running $\alpha_{\mathrm{s}}$, its running of running $\beta_{\mathrm{s}}$, and the tensor-to-scalar ratio $r$, constraints can be derived on the tensor spectral index $n_{\mathrm{t}}$, its running $\alpha_{\mathrm{t}}$, its running of running $\beta_{\mathrm{t}}$, and its running of running of running $\gamma_{\mathrm{t}}$. Using current bounds from the Planck 2015 and BICEP2 datasets and under the slow condition we found the following constraints at 95\% C.L.: $n_{\mathrm{t}}>-0.0157, \alpha_{\mathrm{t}}=-0.00018_{-0.00024}^{+0.00019}, \beta_{\mathrm{t}}=0.00004_{-0.00013}^{+0.00022}$, and $\gamma_{\mathrm{t}}=0.00017_{-0.00019}^{+0.00040}$. Future measurements of the tensor spectrum could therefore be used to test these bounds and the slow-roll condition.
\end{abstract}

DOI: $10.1103 /$ PhysRevD.99.123522

\section{INTRODUCTION}

The detection of $B$-modes in the cosmic microwave background $(\mathrm{CMB})$ polarization angular spectra represents one of the primary goals of modern cosmology. $B$-modes on large angular scales ${ }^{1}$ are indeed produced by the presence of primordial gravitational waves, and their detection can, therefore, provide evidence, albeit indirect, for their existence (see e.g., [1-4]). Several inflationary models, indeed, predict a sizable background of primordial gravitational waves, and its detection could therefore also prove significant information on the physics of inflation (see e.g., [5] for a recent review) and more (see e.g., [6]).

Analyses of the most recent CMB data have provided no evidence for large-scale $B$-modes and bounded the tensor contribution to $r_{0.002}<0.064$ at $95 \%$ C.L. where $r_{0.002}$ is the tensor-over-scalar ratio at spatial wavelength of $k=$ $0.002 \mathrm{Mpc}^{-1}$ [7]. In performing this kind of analysis, it is assumed that the tensor fluctuations follow a primordial spectrum of a power law form as

$$
\mathcal{P}_{\mathrm{t}}(k)=A_{\mathrm{t}} k^{n_{\mathrm{t}}},
$$

where $A_{\mathrm{t}}$ is the primordial tensor amplitude and $n_{\mathrm{t}}$ is the tensor spectral index assumed as scale independent.

\footnotetext{
*william.giare@uniroma1.it

†eleonora.divalentino@manchester.ac.uk

*alessandro.melchiorri@roma1.infn.it

${ }^{1}$ On smaller angular scales gravitational lensing from the intervening dark matter fluctuations generates $B$-modes at later epochs. Several experiments have already detected these modes.
}

In most of the recent analyses, the consistency relation $n_{\mathrm{t}}=-r / 8$ (see e.g., $[8,9]$ and references therein) motivated by standard slow-roll single field inflation is further assumed, leaving $r$ as a single free parameter. However, Eq. (1) is clearly an approximation since $n_{\mathrm{t}}$ is scale dependent and a further parametrization that includes a running and a running of running of tensor modes could be considered.

In this paper we indeed consider a broader set of inflationary parameters, we derive relations between these parameters that are valid under the slow-roll condition, ${ }^{2}$ and we finally compare these relations with current observations. In the next section we describe the inflationary parameters considered, and we show the relevant equations that connect them under the slow-roll condition. In Sec. III we describe our analysis method. In Sec. IV we discuss our results, and in Sec. V we present our conclusions.

\section{INFLATIONARY PARAMETERS}

As stated in the Introduction, in this section we are going to derive some consistency relations among the inflationary parameters that must be satisfied by every single field slowroll inflation model. We will first derive three consistency relations among the scalar and tensor spectral parameters of interest for our analysis, but then we will also generalize our results to higher order parameters proving that, for the single field slow-roll inflationary models, one can obtain as

\footnotetext{
${ }^{2}$ There are several inflationary models that do not satisfy the slow-roll condition; see e.g., [10].
} 
many consistency relations as he wants. However, before deriving these relations, we introduce the following extended parametrization for the primordial spectra of scalar and tensor perturbations:

$$
\begin{gathered}
\mathcal{P}_{\mathrm{S}}(k)=A_{\mathrm{S}}\left(\frac{k}{k_{*}}\right)^{n_{\mathrm{s}}-1+\frac{1}{2} \alpha_{\mathrm{s}} \log \left(k / k_{*}\right)+\frac{1}{6} \beta_{\mathrm{s}}\left(\log \left(k / k_{*}\right)\right)^{2},} \\
\mathcal{P}_{\mathrm{t}}(k)=A_{\mathrm{t}}\left(\frac{k}{k_{*}}\right)^{n_{\mathrm{t}}+\frac{1}{2} \alpha_{\mathrm{t}} \log \left(k / k_{*}\right)+\frac{1}{6} \beta_{\mathrm{t}}\left(\log \left(k / k_{*}\right)\right)^{2},}
\end{gathered}
$$

where $A_{\mathrm{s}}\left(A_{\mathrm{t}}\right)$ is the scalar (tensor) amplitude, $n_{\mathrm{s}}\left(n_{\mathrm{t}}\right)$ is the scalar (tensor) spectral index while $\alpha_{\mathrm{s}}\left(\alpha_{\mathrm{t}}\right)$ and $\beta_{\mathrm{s}}\left(\beta_{\mathrm{t}}\right)$ are the scalar (tensor) spectral index running and the running of running, respectively. The spectral parameters are defined as

$$
\begin{gathered}
n_{\mathrm{s}}-1=\left.\frac{d \log \mathcal{P}_{\mathrm{s}}}{d \log k}\right|_{k=k_{*}}, \\
n_{\mathrm{t}}=\left.\frac{d \log \mathcal{P}_{\mathrm{t}}}{d \log k}\right|_{k=k_{*}}, \\
\alpha_{\mathrm{s}, \mathrm{t}}=\left.\frac{\mathrm{d} n_{\mathrm{s}, \mathrm{t}}}{\mathrm{d} \log k}\right|_{k=k_{*}}, \\
\beta_{\mathrm{s}, \mathrm{t}}=\left.\frac{\mathrm{d} \alpha_{\mathrm{s}, \mathrm{t}}}{\mathrm{d} \log k}\right|_{k=k_{*}}
\end{gathered}
$$

In this work all these relations are to be considered calculated at the pivot scale $k_{*}=0.05 \mathrm{Mpc}^{-1}$, unless otherwise stated.

\section{A. Consistency relations}

One of the most interesting aspects of the inflation theory [11-24] is that, in the framework of the single field slowroll approximation [25-28], from the theory of the quantum inflationary fluctuations [29-45], one can calculate the primordial spectra predicted by inflation ${ }^{3}$ to obtain

$$
\begin{gathered}
\mathcal{P}_{\mathrm{s}}=\left(\frac{1}{8 \pi^{2} M_{p}^{2}}\right)\left(\frac{H^{2}}{\epsilon_{1}}\right)=\left(\frac{1}{12 \pi^{2} M_{p}^{6}}\right)\left(\frac{V^{3}}{V_{\phi}^{2}}\right), \\
\mathcal{P}_{\mathrm{t}}=\left(\frac{2}{\pi^{2} M_{p}^{2}}\right) H^{2}=\left(\frac{2}{3 \pi^{3} M_{p}^{4}}\right) V,
\end{gathered}
$$

where $V$ is the potential of the inflaton scalar field $\phi, V_{\phi}$

\begin{tabular}{|c|c|}
\hline Parameter & Definition \\
\hline \multicolumn{2}{|r|}{ Inflation physics } \\
\hline$\phi$ & Inflaton field \\
\hline$V(\phi)$ & Inflaton potential \\
\hline$a$ & Scale factor \\
\hline$X_{\phi}$ & Partial derivative with respect to $\phi$ \\
\hline$M_{\mathrm{p}}$ & $\begin{array}{l}\text { Reduced Planck mass } \\
\qquad\left(=2.435 \times 10^{18} \mathrm{GeV}\right)\end{array}$ \\
\hline$N=-H d t$ & Number of $e$-folding \\
\hline$\epsilon_{V}=\frac{M_{\mathrm{pl}}^{2} V_{\phi}^{2}}{2 V^{2}}$ & First slow-roll parameter for $V(\phi)$ \\
\hline$\eta_{V}=\frac{M_{\mathrm{pl}}^{2} V_{\phi \phi}}{V}$ & Second slow-roll parameter for $V(\phi)$ \\
\hline$\xi_{V}^{2}=\frac{M_{\mathrm{pl}}^{4} V_{\phi} V_{\phi \phi \phi}}{V^{2}}$ & Third slow-roll parameter for $V(\phi)$ \\
\hline$\varpi_{V}^{3}=\frac{M_{\mathrm{pl}}^{6} V_{\phi}^{2} V_{\phi \phi \phi \phi}}{V^{3}}$ & Fourth slow-roll parameter for $V(\phi)$ \\
\hline$\epsilon_{1}=-\frac{\dot{H}}{H^{2}}=-\frac{1}{2} \frac{d \log \left(H^{2}\right)}{d \log k}$ & First Hubble parameter \\
\hline$\epsilon_{n}=\frac{d \log \left(\epsilon_{n-1}\right)}{d \log k}$ & $n$th Hubble parameter \\
\hline \multicolumn{2}{|r|}{$\Lambda \mathrm{CDM}$ model } \\
\hline$\Omega_{\mathrm{b}} h^{2}$ & Baryon energy density \\
\hline$\Omega_{\mathrm{c}} h^{2}$ & Cold dark matter energy density \\
\hline$\theta_{\mathrm{MC}}$ & $\begin{array}{l}\text { Angular size of the horizon at the last } \\
\text { scattering surface }\end{array}$ \\
\hline$\tau$ & Optical depth \\
\hline $\log \left(10^{10} A_{\mathrm{s}}\right)$ & $\begin{array}{l}\text { Amplitude of primordial scalar } \\
\text { perturbation }\end{array}$ \\
\hline$n_{\mathrm{s}}=d \log \mathcal{P}_{\mathrm{s}} / d \log k$ & Scalar spectral index \\
\hline \multicolumn{2}{|c|}{ Other inflationary parameters } \\
\hline$\alpha_{\mathrm{s}}=\mathrm{d} n_{\mathrm{s}} / \mathrm{d} \log k$ & Scalar spectral index running \\
\hline$\beta_{\mathrm{s}}=\mathrm{d} \alpha_{\mathrm{s}} / \mathrm{d} \log k$ & $\begin{array}{l}\text { Scalar spectral index running of } \\
\text { running }\end{array}$ \\
\hline$r=\mathcal{P}_{\mathrm{t}} / \mathcal{P}_{\mathrm{s}}$ & Tensor-to-scalar ratio \\
\hline$n_{\mathrm{t}}=d \log \mathcal{P}_{\mathrm{t}} / d \log k$ & Tensor spectral index \\
\hline$\alpha_{\mathrm{t}}=\mathrm{d} n_{\mathrm{t}} / \mathrm{d} \log k$ & Tensor spectral index running \\
\hline$\beta_{\mathrm{t}}=\mathrm{d} \alpha_{\mathrm{t}} / \mathrm{d} \log k$ & $\begin{array}{l}\text { Tensor spectral index running of } \\
\text { running }\end{array}$ \\
\hline$\gamma_{\mathrm{t}}=\mathrm{d} \beta_{\mathrm{t}} / \mathrm{d} \log k$ & $\begin{array}{l}\text { Tensor spectral index running of } \\
\text { running of running }\end{array}$ \\
\hline
\end{tabular}
indicates the derivative with respect to $\phi$, and $\epsilon_{1}$ is the first

\footnotetext{
${ }^{3} \mathrm{We}$ are considering the curvature perturbation to be generated by the quantum fluctuations of a slowly rolling inflaton field. Other different theories predict curvature perturbations generated by quantum fluctuations of fields that have nothing to do with the inflation; see e.g., [46].
}

TABLE I. Conventions and definitions.

Hubble parameter (See Table I). Since in the slow-roll limit the logarithm derivative $\frac{d}{d \ln k}=-\frac{d}{d N}=\frac{1}{H} \frac{d}{d t}$ can be written in terms of $V_{\phi}$ as $\frac{d}{d \ln k} \approx-M_{p}^{2} \frac{V_{\phi}}{V} \frac{d}{d \phi}$, the scalar and tensor spectral parameters can be calculated in terms of the slowroll parameters $\epsilon_{V}, \eta_{V}, \xi_{V}^{2}$, and $\varpi_{V}^{3}$ defined in Table I. For the scalar parameters we have [47]

$$
\begin{gathered}
n_{\mathrm{s}}-1=2 \eta_{V}-6 \epsilon_{V}, \\
\alpha_{\mathrm{s}}=+16 \epsilon_{V} \eta_{V}-24 \epsilon_{V}^{2}-2 \xi_{V}^{2},
\end{gathered}
$$

\footnotetext{
${ }^{4}$ We are at the horizon crossing where $k=a H$ and therefore $d \log k=d \log a+d \log H=H d t+\frac{\dot{H}}{H} d t \approx H d t=-d N$ by the slow-roll condition $\frac{\dot{H}}{H} \ll 1$.
} 


$$
\begin{aligned}
\beta_{\mathrm{S}}= & -192 \epsilon_{V}^{3}+192 \epsilon_{V}^{2} \eta_{V}-32 \epsilon_{V} \eta_{V}^{2}-24 \epsilon_{V} \xi_{V}^{2} \\
& +2 \eta_{V} \xi_{V}^{2}+2 \varpi_{V}^{3} .
\end{aligned}
$$

On the other hand, for the tensor parameters we obtain

$$
\begin{gathered}
n_{\mathrm{t}}=-2 \epsilon_{V}, \\
\alpha_{\mathrm{t}}=+4 \epsilon_{V} \eta_{V}-8 \epsilon_{V}^{2}, \\
\beta_{t}=-64 \epsilon_{V}^{3}-8 \epsilon_{V} \eta_{V}^{2}-4 \epsilon_{V} \xi_{V}^{2}+56 \epsilon_{V}^{2} \eta_{V} .
\end{gathered}
$$

Once again, all these equations have to be considered evaluated when the inflaton field $\phi=\phi_{\star}$ where the mode crosses the Hubble radius for the first time: $k_{\star}=a_{\star} H_{\star}$.

The single field slow-roll paradigm implies the following well known consistency relation $[8,9,48-54]$ :

$$
r \equiv \frac{\mathcal{P}_{\mathrm{t}}\left(k_{*}\right)}{\mathcal{P}_{\mathrm{s}}\left(k_{*}\right)}=-8 n_{\mathrm{t}}
$$

Two other interesting consistency relations can easily be obtained reversing Eqs. (13), (14), and (15) and finding the expressions of the slow-roll parameters in terms of the tensor parameters: $\epsilon_{V}=-\frac{1}{2} n_{\mathrm{t}}, \eta_{V}=-n_{\mathrm{t}}-\frac{1}{2} \frac{\alpha_{\mathrm{t}}}{n_{\mathrm{t}}}$, and $\xi_{V}^{2}=n_{\mathrm{t}}^{2}+\frac{3}{2} \alpha_{\mathrm{t}}-\frac{1}{2}\left(\frac{\alpha_{\mathrm{t}}}{n_{\mathrm{t}}}\right)^{2}+\frac{1}{2}\left(\frac{\beta_{\mathrm{t}}}{n_{\mathrm{t}}}\right)$. Substituting these relations into Eqs. (10) and (11), it is easy to find the following relations among scalar and tensor parameters (see e.g., [9]):

$$
\begin{gathered}
\alpha_{\mathrm{t}}=n_{\mathrm{t}}^{2}-n_{\mathrm{t}}\left(n_{\mathrm{s}}-1\right), \\
\beta_{\mathrm{t}}=n_{\mathrm{t}}\left(\alpha_{\mathrm{t}}-\alpha_{\mathrm{s}}\right)+\frac{\alpha_{\mathrm{t}}^{2}}{n_{\mathrm{t}}} .
\end{gathered}
$$

Using the consistency relation (16), one can see that all the tensor spectral parameters can be expressed in terms of the tensor-to-scalar ratio $r$, the spectral index $n_{s}$, and the scalar running $\alpha_{s}$ as follows:

$$
n_{\mathrm{t}}=-\frac{r}{8}
$$

$$
\begin{gathered}
\alpha_{\mathrm{t}}=\frac{r}{8}\left(n_{s}-1\right)+\frac{r^{2}}{64}, \\
\beta_{\mathrm{t}}=\frac{r}{8}\left[\alpha_{\mathrm{s}}-\left(n_{\mathrm{s}}-1\right)^{2}\right]-\frac{3 r^{2}}{64}\left(n_{\mathrm{s}}-1\right)-\frac{r^{3}}{256} .
\end{gathered}
$$

\section{B. Higher order generalization}

The previous consistency relations can be derived in a more elegant way using a slightly different version of the slow-roll parameters that we may call Hubble parameters, $\left\{\epsilon_{1} \cdots \epsilon_{n}\right\}$ defined in such a way that

$$
\begin{aligned}
\epsilon_{1} & =-\frac{1}{2} \frac{d \log \left(H^{2}\right)}{d \log k}=-\frac{\dot{H}}{H^{2}}, \\
\epsilon_{2} & =\frac{1}{\epsilon_{1}} \frac{d \epsilon_{1}}{d \log k}=\frac{d \log \left(\epsilon_{1}\right)}{d \log k}, \\
& \ldots \\
\epsilon_{n} & =\frac{d \log \left(\epsilon_{n-1}\right)}{d \log k} .
\end{aligned}
$$

Using these parameters we can prove a quite general result: in the single field slow-roll inflation, if we fix the scalar parameters up to the scalar running $\frac{d^{n-1} n_{\mathrm{s}}}{d(\log k)^{n-1}}$, we immediately fix all the tensor spectral parameters up to the tensor running $\frac{d^{n} n_{\mathrm{t}}}{d(\log k)^{n}}$ proving that, for the single field slow-roll inflation, one can obtain as many relations as one wants. The scalar and tensor spectral indices can easily be calculated in terms of the Hubble parameters (22):

$$
\begin{gathered}
n_{\mathrm{s}}-1=\frac{d \log \mathcal{P}_{\mathrm{s}}}{d \log k}=\frac{d \log H^{2}}{d \log k}-\frac{d \log \epsilon_{1}}{d \log k}=-2 \epsilon_{1}-\epsilon_{2}, \\
n_{\mathrm{t}}=\frac{d \log \mathcal{P}_{\mathrm{t}}}{d \log k}=\frac{d \log H^{2}}{d \log k}=-2 \epsilon_{1} .
\end{gathered}
$$

It is therefore easy to convince yourself that in the scalar case the first $n$ scalar parameters will be (regular) functions of the first $n+1$ Hubble parameters:

$$
\begin{aligned}
n_{\mathrm{s}}-1 & =-2 \epsilon_{1}-\epsilon_{2} \equiv f_{1}\left(\epsilon_{1}, \epsilon_{2}\right), \\
\frac{d n_{\mathrm{s}}}{d \log k} & \equiv \alpha_{\mathrm{s}}=-2 \epsilon_{1} \epsilon_{2}-\epsilon_{2} \epsilon_{3} \equiv f_{2}\left(\epsilon_{1}, \epsilon_{2}, \epsilon_{3}\right), \\
\frac{d^{2} n_{\mathrm{s}}}{d(\log k)^{2}} & \equiv \beta_{\mathrm{s}}=-2 \epsilon_{1} \epsilon_{2}^{2}-2 \epsilon_{1} \epsilon_{2} \epsilon_{3}-\epsilon_{2} \epsilon_{3}^{2}-\epsilon_{2} \epsilon_{3} \epsilon_{4} \equiv f_{3}\left(\epsilon_{1}, \epsilon_{2}, \epsilon_{3}, \epsilon_{4}\right), \\
& \ldots \\
\frac{d^{n-1} n_{\mathrm{s}}}{d(\log k)^{n-1}} & =f_{n}\left(\epsilon_{1}, \ldots, \epsilon_{n+1}\right) .
\end{aligned}
$$

In the tensor case, the first $n+1$ tensor parameters will be (regular) functions of the first $n+1$ Hubble parameters: 


$$
\begin{aligned}
n_{\mathrm{t}} & =-2 \epsilon_{1} \equiv g_{1}\left(\epsilon_{1}\right), \\
\frac{d n_{\mathrm{t}}}{d \log k} & \equiv \alpha_{\mathrm{t}}=-2 \epsilon_{1} \epsilon_{2} \equiv g_{2}\left(\epsilon_{1}, \epsilon_{2}\right), \\
\frac{d^{2} n_{\mathrm{t}}}{d(\log k)^{2}} & \equiv \beta_{\mathrm{t}}=-2 \epsilon_{1} \epsilon_{2}^{2}-2 \epsilon_{1} \epsilon_{2} \epsilon_{3} \equiv g_{3}\left(\epsilon_{1}, \epsilon_{2}, \epsilon_{3}\right), \\
\frac{d^{3} n_{\mathrm{t}}}{d(\log k)^{3}} & =\frac{d \beta_{\mathrm{t}}}{d \log (k)}=-2 \epsilon_{1} \epsilon_{2}^{3}-6 \epsilon_{1} \epsilon_{2}^{2} \epsilon_{3}-2 \epsilon_{1} \epsilon_{2} \epsilon_{3}^{2}-2 \epsilon_{1} \epsilon_{2} \epsilon_{3} \epsilon_{4} \equiv g_{4}\left(\epsilon_{1}, \epsilon_{2}, \epsilon_{3}, \epsilon_{4}\right), \\
& \cdots \\
\frac{d^{n} n_{\mathrm{t}}}{d(\log k)^{n}} & =g_{n+1}\left(\epsilon_{1}, \ldots, \epsilon_{n+1}\right) .
\end{aligned}
$$

Note that the two sets of functions $\left\{f_{1}, \ldots, f_{n}\right\}$ and $\left\{g_{1}, \ldots, g_{n}\right\}$ are introduced only to render explicit the dependency of the scalar and tensor parameters in terms of the Hubble parameters.

Because of the structure of the tensor runnings (26), it is easy to reverse the equations in such a way that we can express the Hubble parameters $\left\{\epsilon_{1}, \ldots, \epsilon_{n+1}\right\}$ as functions $\left\{\tilde{g}_{1}, \ldots, \tilde{g}_{n}\right\}$ of the tensor parameters $\left\{n_{\mathrm{t}}, \alpha_{\mathrm{t}}, \beta_{\mathrm{t}}, \ldots, \frac{d^{n} n_{\mathrm{t}}}{d(\log k)^{n}}\right\}$ :

$$
\begin{aligned}
\epsilon_{1} & =-\frac{1}{2} n_{\mathrm{t}} \equiv \tilde{g}_{1}\left(n_{t}\right), \\
\epsilon_{2} & =\frac{\alpha_{\mathrm{t}}}{n_{\mathrm{t}}} \equiv \tilde{g}_{2}\left(n_{\mathrm{t}}, \alpha_{\mathrm{t}}\right), \\
\epsilon_{3} & =\frac{\beta_{\mathrm{t}}}{\alpha_{\mathrm{t}}}-\frac{\alpha_{\mathrm{t}}}{n_{\mathrm{t}}} \equiv \tilde{g}_{3}\left(n_{\mathrm{t}}, \alpha_{\mathrm{t}}, \beta_{\mathrm{t}}\right), \\
\epsilon_{4} & =\left(\frac{n_{\mathrm{t}}}{n_{\mathrm{t}} \beta_{\mathrm{t}}-\alpha_{\mathrm{t}}^{2}}\right)\left[\frac{d^{3} n_{\mathrm{t}}}{d(\log k)^{3}}+\frac{\alpha_{\mathrm{t}}^{3}}{n_{\mathrm{t}}^{2}}-\frac{\alpha_{\mathrm{t}} \beta_{\mathrm{t}}}{n_{\mathrm{t}}}-\frac{\beta_{\mathrm{t}}^{2}}{\alpha_{\mathrm{t}}}\right] \equiv \tilde{g}_{4}\left(n_{\mathrm{t}}, \alpha_{\mathrm{t}}, \beta_{\mathrm{t}}, \frac{d^{3} n_{\mathrm{t}}}{d(\log k)^{3}}\right), \\
& \ldots \\
\epsilon_{n+1} & =\tilde{g}_{n+1}\left(n_{\mathrm{t}}, \alpha_{\mathrm{t}}, \beta_{\mathrm{t}}, \frac{d^{3} n_{\mathrm{t}}}{d(\log k)^{3}}, \ldots, \frac{d^{n} n_{\mathrm{t}}}{d(\log k)^{n}}\right) .
\end{aligned}
$$

Substituting in the scalar equations (25) we obtain the following $n$ relations among scalar and tensor parameters:

$$
\begin{aligned}
n_{\mathrm{s}}-1 & =n_{\mathrm{t}}-\frac{\alpha_{\mathrm{t}}}{n_{\mathrm{t}}} \\
\frac{d n_{\mathrm{s}}}{d \log k} & \equiv \alpha_{\mathrm{s}}=\alpha_{\mathrm{t}}+\left(\frac{\alpha_{\mathrm{t}}}{n_{\mathrm{t}}}\right)^{2}-\frac{\beta_{\mathrm{t}}}{n_{\mathrm{t}}} \\
\frac{d^{2} n_{\mathrm{s}}}{d(\log k)^{2}} & \equiv \beta_{\mathrm{s}}=\beta_{\mathrm{t}}-2\left(\frac{\alpha_{\mathrm{t}}^{3}}{n_{\mathrm{t}}^{3}}\right)+3\left(\frac{\alpha_{\mathrm{t}} \beta_{\mathrm{t}}}{n_{\mathrm{t}}^{2}}\right)-\frac{1}{n_{\mathrm{t}}}\left(\frac{d^{3} n_{\mathrm{t}}}{d(\log k)^{3}}\right), \\
\ldots & \\
\frac{d^{n-1} n_{\mathrm{s}}}{d(\log k)^{n-1}} & =\tilde{f}_{n}\left(n_{\mathrm{t}}, \alpha_{\mathrm{t}}, \beta_{\mathrm{t}}, \frac{d^{3} n_{\mathrm{t}}}{d(\log k)^{3}}, \ldots, \frac{d^{n} n_{\mathrm{t}}}{d(\log k)^{n}}\right) .
\end{aligned}
$$

Note that here the tilded functions are nothing other than the untilded functions up to a variables redefinition. However, by Eq. (16), we also know that, for the single field slow-roll inflation, $r=-8 n_{\mathrm{t}}$ and so the set of all the $n+1$ relations is 


$$
\begin{aligned}
r & =-8 n_{\mathrm{t}}, \\
n_{\mathrm{s}}-1 & =n_{\mathrm{t}}-\frac{\alpha_{\mathrm{t}}}{n_{\mathrm{t}}} \\
\frac{d n_{\mathrm{s}}}{d \log k} & \equiv \alpha_{\mathrm{s}}=\alpha_{\mathrm{t}}+\left(\frac{\alpha_{\mathrm{t}}}{n_{\mathrm{t}}}\right)^{2}-\frac{\beta_{\mathrm{t}}}{n_{\mathrm{t}}} \\
\frac{d^{2} n_{\mathrm{s}}}{d(\log k)^{2}} & \equiv \beta_{\mathrm{s}}=\beta_{\mathrm{t}}-2\left(\frac{\alpha_{\mathrm{t}}^{3}}{n_{\mathrm{t}}^{3}}\right)+3\left(\frac{\alpha_{\mathrm{t}} \beta_{\mathrm{t}}}{n_{\mathrm{t}}^{2}}\right)-\frac{1}{n_{\mathrm{t}}}\left(\frac{d^{3} n_{\mathrm{t}}}{d(\log k)^{3}}\right), \\
\ldots & \\
\frac{d^{n-1} n_{\mathrm{s}}}{d(\log k)^{n-1}} & =\tilde{f}_{n}\left(n_{\mathrm{t}}, \alpha_{\mathrm{t}}, \beta_{\mathrm{t}}, \frac{d^{3} n_{\mathrm{t}}}{d(\log k)^{3}}, \ldots, \frac{d^{n} n_{\mathrm{t}}}{d(\log k)^{n}}\right) .
\end{aligned}
$$

Note that if all the left side members of (29) (i.e., $r$ and all the $n$ scalar parameters) are fixed, all the $n+1$ right side tensor parameters are fixed as well. Therefore by the system above, one can calculate how many consistency relations one wants. The first three lines of (29) are nothing other than the three consistency relations (16), (17), and (18) derived in the previous subsection, while the fourth line is another explicit consistency relation that can be put in the form

$$
\frac{d \beta_{\mathrm{t}}}{d \log k} \equiv \gamma_{\mathrm{t}}=n_{\mathrm{t}}\left(\beta_{\mathrm{t}}-\beta_{\mathrm{s}}\right)-2\left(\frac{\alpha_{\mathrm{t}}^{3}}{n_{\mathrm{t}}^{2}}\right)+3\left(\frac{\alpha_{\mathrm{t}} \beta_{\mathrm{t}}}{n_{\mathrm{t}}}\right)
$$

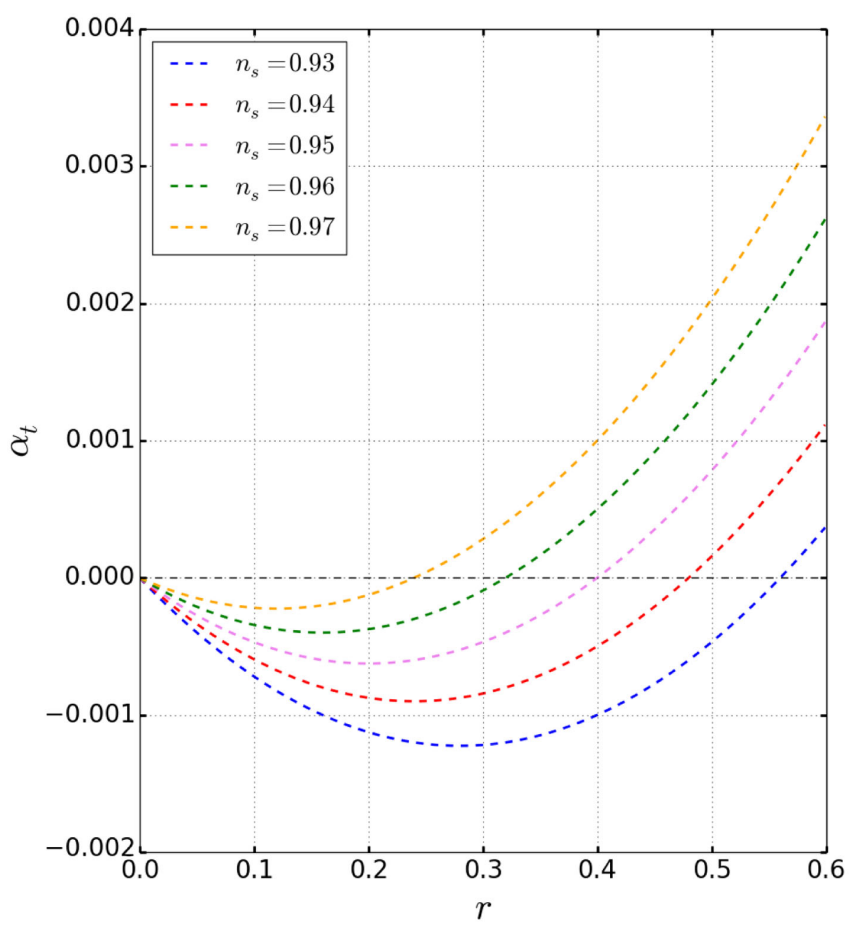

Therefore, in practice, if single field slow-roll inflation is valid, a determination of $r$ and the first $n$ scalar parameters immediately fixes also the first $n+1$ tensor parameters. An independent measurement of these tensor parameters can therefore be used for testing the slow-roll condition. This is what we plan to do in the next two sections of this paper. As we will see, even if the tensor component has not yet been detected, some interesting regions of the parameter space are excluded.

A first point of interest is to investigate the sign of the tensor runnings predicted by the slow-roll paradigm. Of course we expect a red tensor tilt by Eq. (19). More interesting is to investigate the sign of the tensor running $\alpha_{\mathrm{t}}$

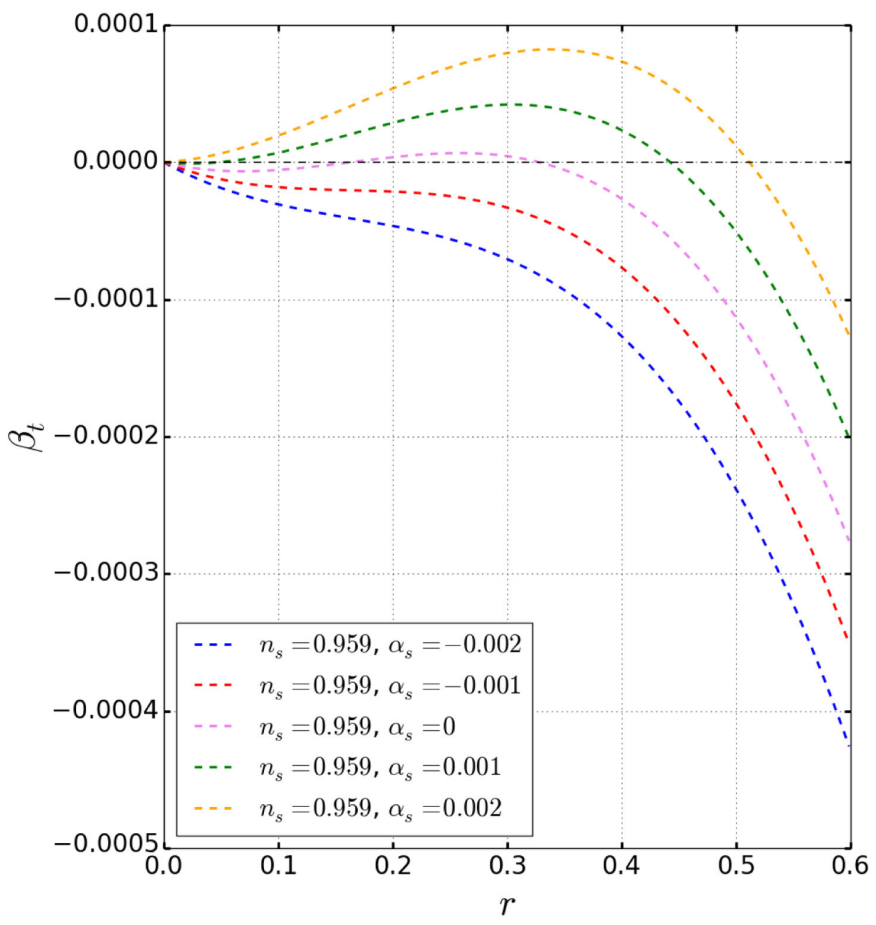

FIG. 1. On the left side panel the consistency relation (20) for different values of $n_{\mathrm{s}}$. On the right side panel the consistency relation (21) for $n_{\mathrm{s}}=0.959$ and the different indicated values of $\alpha_{\mathrm{s}}$. 
and the running of running $\beta_{\mathrm{t}}$. Both of them consist of negative and positive contributions, so the final sign depends on the relative amplitude of these contributions. As regards $\alpha_{\mathrm{t}}$, since from the data we know that $n_{\mathrm{s}}-1<0$ at more than 5 standard deviations, the linear contribution in $r$ is negative while the contribution $\propto r^{2}$ is of course positive. Therefore at sufficiently small $r, \alpha_{\mathrm{t}}$ should be negative. As regards $\beta_{\mathrm{t}}$, the situation is more complicated since the sign of the linear term $\propto \frac{r}{8}\left[\alpha_{\mathrm{s}}-\left(n_{\mathrm{s}}-1\right)^{2}\right]$ depends on the sign and above all on the amplitude of $\alpha_{\mathrm{s}}$ itself. On the other hand, the term $\propto-r^{2}\left(n_{\mathrm{s}}-1\right)$ is positive while the cubic contribution in $r$ is negative and expected to be negligible for small $r$. In Fig. 1 we plot both the consistency relation (20) and the consistency relation (21) for different values of the scalar parameters, exploring a range $r \in[0.001,0.6]$. On the left side panel of Fig. 1, we can see how the tensor running $\alpha_{\mathrm{t}}$ is always negative at small $r$ as expected. Depending on the value of $n_{\mathrm{s}}$, it changes sign at different $r$ as large as $n_{\mathrm{s}}$ is small. Since $n_{\mathrm{S}}$ is well constrained to be $n_{\mathrm{s}} \sim 0.96$, a positive scalar running for the slow-roll paradigm inflation would imply a value of $r \gtrsim 0.3$ that seems to be excluded by the present data. On the right side panel of the same figure we can instead see how $\alpha_{\mathrm{s}}$, even if small of order $10^{-3}$, significantly alters the final sign of $\beta_{\mathrm{t}}$ : if we consider a small positive scalar running, for sufficiently small $r, \beta_{\mathrm{t}}$ will be positive, while considering a small negative scalar running, $\beta_{\mathrm{t}}$ will always be negative. For $\alpha_{\mathrm{s}} \simeq 0$ the sign of $\beta_{\mathrm{t}}$ changes three times at different $r$, being negative at very small $r$ where the negative linear term $\propto-r\left(n_{\mathrm{s}}-1\right)^{2}$ is dominant as expected.

\section{METHOD}

We perform a Monte Carlo Markov chain (MCMC) analysis using the November 2016 version of the publicly available package COSMOMC [55], with a convergence diagnostic based on the Gelman and Rubin statistic. This includes the support for the Planck data release 2015 Likelihood Code [56] (see http://cosmologist.info/ cosmomc/) and implements an efficient sampling by using the fast/slow parameter decorrelations [57]. We also vary the foreground parameters as described in [56,58].

We consider the six parameters of the standard $\Lambda$ CDM model, i.e., the baryon $\omega_{\mathrm{b}} \equiv \Omega_{\mathrm{b}} h^{2}$ and cold dark matter $\omega_{\mathrm{c}} \equiv \Omega_{\mathrm{c}} h^{2}$ energy densities, the angular size of the horizon at the last scattering surface $\theta_{\mathrm{MC}}$, the optical depth $\tau$, the amplitude of primordial scalar perturbation $\log \left(10^{10} A_{\mathrm{s}}\right)$, and the scalar spectral index $n_{\mathrm{s}}$. We add the scalar spectral index running $\alpha_{\mathrm{s}}$, the scalar running of running $\beta_{\mathrm{s}}$, the tensor-to-scalar ratio $r$, the tensor spectral index $n_{\mathrm{t}}$, the tensor spectral index running $\alpha_{\mathrm{t}}$, and the tensor running of running $\beta_{\mathrm{t}}$. We normalize the inflationary parameters to the pivot wavelength $k_{*}=0.05 \mathrm{Mpc}^{-1}$. Therefore, for our theoretical baseline we consider 12 parameters that we
TABLE II. List of the external flat priors on the cosmological parameters assumed in this paper.

\begin{tabular}{lc}
\hline \hline Parameter & Prior \\
\hline$\Omega_{\mathrm{b}} h^{2}$ & {$[0.005,0.1]$} \\
$\Omega_{\mathrm{c}} h^{2}$ & {$[0.001,0.99]$} \\
$100 \theta_{\mathrm{MC}}$ & {$[0.5,10]$} \\
$\tau$ & {$[0.01,0.8]$} \\
$\log \left(10^{10} A_{s}\right)$ & {$[2,4]$} \\
$n_{s}$ & {$[0.8,1.2]$} \\
$\alpha_{s}$ & {$[-0.1,0.1]$} \\
$\beta_{s}$ & {$[-0.1,0.1]$} \\
$r$ & {$[0.001,3]$} \\
$n_{t}$ & {$[-2,0]$} \\
$\alpha_{t}$ & {$[-0.4,0.4]$} \\
$\beta_{t}$ & {$[-0.4,0.4]$} \\
\hline \hline
\end{tabular}

vary in a range of external, conservative priors listed in Table II. We stress that for $r$ we have decided to consider a prior lower limit $r>0.001$ (roughly corresponding to the chain sampling). This is because the current data do not lead to a detection of a nonvanishing $r$, and so any value of $n_{\mathrm{t}}, \alpha_{\mathrm{t}}$, and $\beta_{\mathrm{t}}$ would give a good fit as long as $r$ is close enough to zero. So without the lower limit above we could not say anything about the tensor spectral parameters.

Our reference datasets are based on CMB temperature and polarization anisotropies. We analyze the BB power spectrum as measured by the Joint Analysis of BICEP2/ Keck Array and Planck (BKP) collaborations [59], in combination with the temperature and polarization Planck 2015 likelihood [56]. More precisely, we use the TT and the TT, TE, EE high- $\ell$ likelihood together with the TEB pixel-based low- $\ell$ likelihood and the BB power spectrum polarization data from the BKP collaborations.

\section{RESULTS}

In this section we present the results of our MCMC analysis. We first present the constraints on all the parameters considered in our cosmological model, without assuming the consistency relations, i.e., deriving the bounds on the tensor parameters directly from the data. As a second step we compare the constraints on the tensor parameters with those derived assuming the slow-roll conditions described in Sec. II.

\section{A. Parameter constraints without assuming the slow-roll condition}

In Table III we present the constraints at 68\% C.L. (or the upper/lower limits at $95 \%$ C.L.) for different datasets from the Planck 2015 temperature and polarization data together with the BB power spectrum polarization as measured from the BKP collaboration. A first point of interest is to investigate the impact of the inclusion of the scalar and tensor parameters $r, n_{\mathrm{t}}, \alpha_{\mathrm{s}, \mathrm{t}}$, and $\beta_{\mathrm{s}, \mathrm{t}}$ on the remaining six 
TABLE III. Constraints on all the parameters of the $\Lambda \mathrm{CDM}+\alpha_{\mathrm{s}}+\beta_{\mathrm{s}}+r+n_{\mathrm{t}}+\alpha_{\mathrm{t}}+\beta_{\mathrm{t}}$ model for different datasets. For each dataset we quote the $68 \%$ C.L. constraint or the $95 \%$ C.L. upper/lower limit.

\begin{tabular}{lcccc}
\hline \hline Parameter & TT + low P & TT, TE, EE + low P & TT + low P + BKP & TT, TE, EE, +low P + BKP \\
\hline$\Omega_{\mathrm{b}} h^{2}$ & $0.02214 \pm 0.00033$ & $0.02220 \pm 0.00018$ & $0.02209_{-0.00030}^{+0.0035}$ & $0.02218 \pm 0.00018$ \\
$\Omega_{\mathrm{c}} h^{2}$ & $0.1207 \pm 0.0024$ & $0.1203 \pm 0.0015$ & $0.1210 \pm 0.0024$ & $0.1206 \pm 0.0016$ \\
$100 \theta_{\mathrm{MC}}$ & $1.04079 \pm 0.00050$ & $1.04072 \pm 0.00032$ & $1.04076 \pm 0.00048$ & $1.04071 \pm 0.00033$ \\
$\tau$ & $0.092 \pm 0.023$ & $0.094_{-0.018}^{+0.020}$ & $0.092 \pm 0.023$ & $0.094 \pm 0.019$ \\
$\log \left(10^{10} A_{\mathrm{s}}\right)$ & $3.120 \pm 0.046$ & $3.123 \pm 0.037$ & $3.120 \pm 0.045$ & $3.123 \pm 0.038$ \\
$n_{\mathrm{s}}$ & $0.9590 \pm 0.0079$ & $0.9598 \pm 0.0056$ & $0.9579 \pm 0.0077$ & $0.9586 \pm 0.0057$ \\
$\alpha_{\mathrm{s}}$ & $0.007 \pm 0.014$ & $0.007 \pm 0.011$ & $0.010 \pm 0.014$ & $0.009 \pm 0.011$ \\
$\beta_{\mathrm{s}}$ & $0.028 \pm 0.016$ & $0.027 \pm 0.014$ & $0.030 \pm 0.016$ & $0.028 \pm 0.014$ \\
$r$ & $<0.785$ & $<0.629$ & $<0.127$ & $<0.124$ \\
$n_{\mathrm{t}}$ & $>-0.890$ & $>-0.907$ & $>-0.990$ & $>-1.15$ \\
$\alpha_{\mathrm{t}}$ & $<0.209$ & $<0.186$ & $<0.231$ & $<0.232$ \\
$\beta_{\mathrm{t}}$ & $>-0.0707$ & $>-0.0667$ & $>-0.102$ & $>-0.0918$ \\
\hline \hline
\end{tabular}

parameters of the standard $\Lambda \mathrm{CDM}$ model. Comparing our results with those obtained from the Planck collaboration in Ref. [60], we can see that there are no significant shifts on the $\Lambda$ CDM parameters. The largest deviations with respect to the standard scenario are present in the spectral index $n_{\mathrm{s}}$ that is $\sim 0.8(\sim 1)$ standard deviations lower for both the Planck TT + low $\mathrm{P}(+\mathrm{BKP})$ and the Planck TT, TE, EE, + low $\mathrm{P}(+\mathrm{BKP})$ datasets. The optical depth $\tau$ is instead $\sim 0.6$ standard deviations higher as well as the scalar amplitude $\log \left(10^{10} A_{\mathrm{s}}\right)$ that is about $\sim 0.7 / 0.8$ standard deviations higher (depending on the datasets).

As concerns the scalar spectral parameters, we can see that the data provide an indication for $\beta_{\mathrm{s}}>0$ at about 2 standard deviations for all the datasets. Similar results were found in [61] analyzing models with only the scalar spectral parameters allowed and also in [62] analyzing tensor models assuming scale at independent $n_{\mathrm{t}}$. Therefore one can conclude that the inclusion of the tensor spectral parameters does not alter significantly the bounds on the scalar parameters as well as the bounds on the other standard $\Lambda$ CDM parameters.

On the other hand, for the tensor parameters, we can see that considering the BKP data together with the Planck data we can obtain tighter bounds on the tensor-to-scalar ratio $r$, while the bounds on the scalar parameters are of the same order. Therefore in the following discussion we will focus on the two datasets Planck TT + low TEB + BKP and Planck TT TE EE + low TEB + BKP. For the tensor-toscalar ratio we obtain $r<0.127$ (95\% C.L. Planck TT + low TEB + BKP) and $r<0.124$ (95\% C.L. Planck TT TE EE + low TEB + BKP).

As concerns the tensor index $n_{\mathrm{t}}$ in our analysis we restrict our attention to red tensor tilts (i.e., $n_{\mathrm{t}}<0$ ) and we obtain the two lower bounds $n_{\mathrm{t}}>-0.990$ (95\% C.L. Planck TT + low TEB + BKP) and $n_{\mathrm{t}}>-1.15$ (95\% C.L. Planck TT TE EE + low TEB + BKP).
For the tensor running $\alpha_{\mathrm{t}}$ and the running of running $\beta_{\mathrm{t}}$ we instead fix a prior sampling range $[-0.4,0.4]$ for both the parameters, as we can see in Table II. For $\alpha_{\mathrm{t}}$ we have $\alpha_{\mathrm{t}}<0.231$ (95\% C.L. Planck TT + low TEB + BKP) and $\alpha_{\mathrm{t}}<0.232(95 \%$ C.L. Planck TT TE EE + low TEB + BKP) while for $\beta_{\mathrm{t}}$ we have $\beta_{\mathrm{t}}>-0.102$ (95\% C.L. Planck $\mathrm{TT}+$ low TEB + BKP) and $\beta_{\mathrm{t}}>-0.0918 \quad(95 \% \quad$ C.L. Planck TT TE EE + low TEB + BKP).

\section{B. Constraints on tensor parameters assuming slow roll}

We now derive the constraints on the tensor parameters $n_{\mathrm{t}}, \alpha_{\mathrm{t}}$, and $\beta_{\mathrm{t}}$ from the observed constraints on $n_{\mathrm{s}}, \alpha_{\mathrm{s}}$, and $r$ reported in the previous subsection assuming the relations described in Sec. II under the slow-roll condition. Moreover, we can check the slow-roll approximation by comparing these results with the model independent constraints reported in the previous subsection. Using the consistency relations one expects that the bounds on the tensor parameters drastically shrink since, as explained in Sec. II, all the tensor parameters are related to $r$ and the scalar parameters.

Imposing the consistency relations, for the tensor tilt we find $n_{\mathrm{t}}>-0.0158(95 \%$ C.L., Planck TT + low TEB + $\mathrm{BKP})$ and $n_{\mathrm{t}}>-0.0157$ (95\% C.L., Planck TT TE $\mathrm{EE}+$ low TEB + BKP).

In the left side panel of Fig. 2 we plot the marginalized joint confidence contours at $68 \%$ and $95 \%$ C.L. in the plane $\left(r, n_{\mathrm{t}}\right)$ while in the right side panel of the same figure we plot the marginalized contours with $n_{\mathrm{t}}$ derived by the consistency relation (19). As one can see, assuming the consistency relation (19), to each $r$ corresponds one fixed $n_{t}$. The constraints obtained relaxing the consistency relations are of course embedded into the contours for the relaxed parameters. As concerns the tensor runnings, for $\alpha_{\mathrm{t}}$ the limits obtained imposing the consistency relations become $\alpha_{\mathrm{t}}=-0.00018_{-0.00025}^{+0.00019} \quad(95 \%$ C.L., 

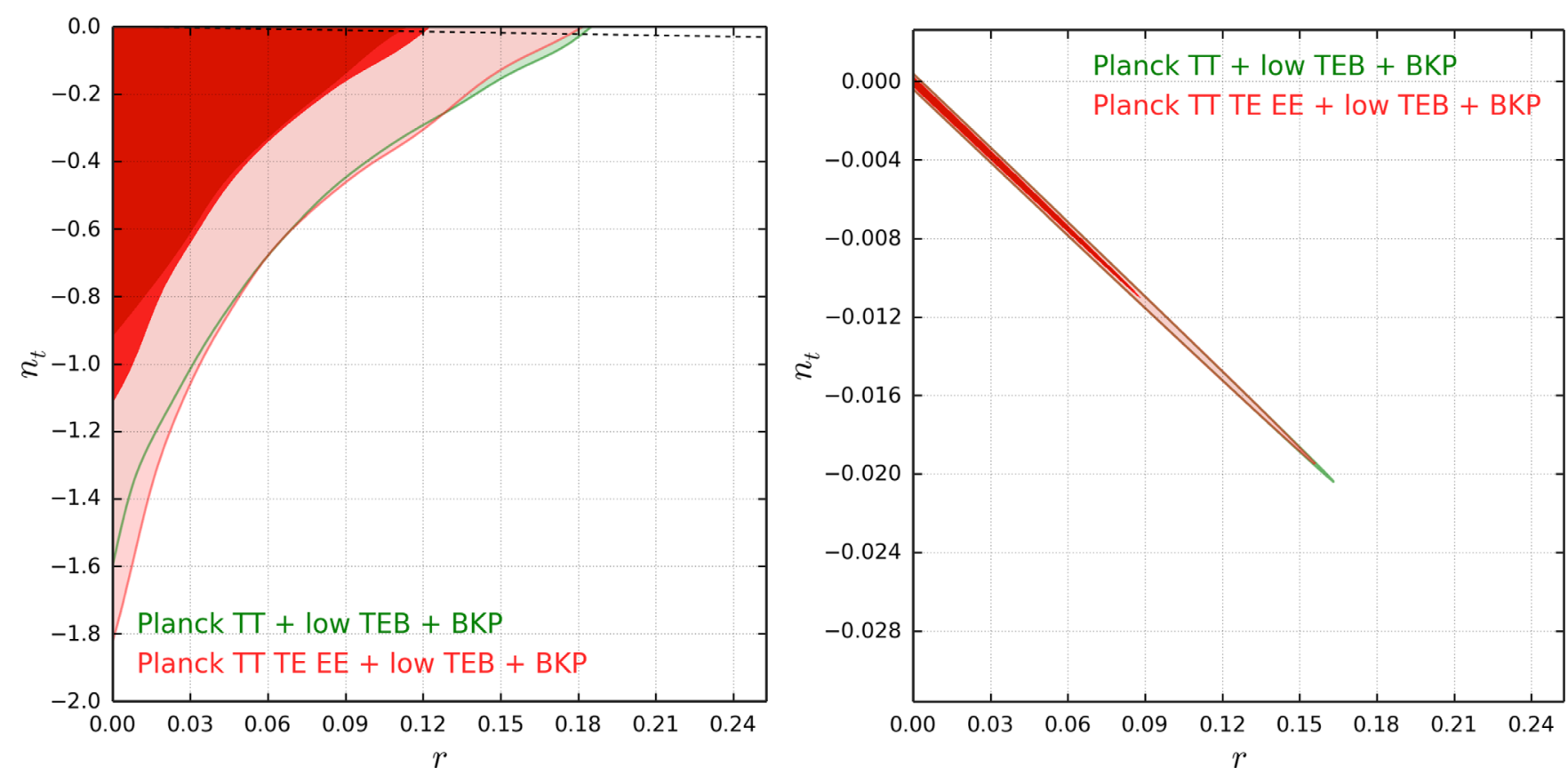

FIG. 2. Marginalized joint confidence contours at $68 \%$ and $95 \%$ C.L. in the plane $\left(n_{\mathrm{t}}, r\right)$ for the indicated datasets. On the left (right) side panel the contours obtained relaxing (imposing) the consistency relations. The black dashed line in the left panel represents the consistency relation $n_{\mathrm{t}}=-\frac{r}{8}$.

Planck TT + low TEB + BKP) and $\alpha_{\mathrm{t}}=-0.00018_{-0.00024}^{+0.00019}$ (95\% C.L., Planck TT TE EE + low TEB + BKP), while for $\beta_{\mathrm{t}}$ we find $\beta_{\mathrm{t}}=0.00004_{-0.00019}^{+0.00027}$ (95\% C.L., Planck TT + low TEB + BKP) and $\beta_{\mathrm{t}}=0.00004_{-0.00013}^{+0.00022}$

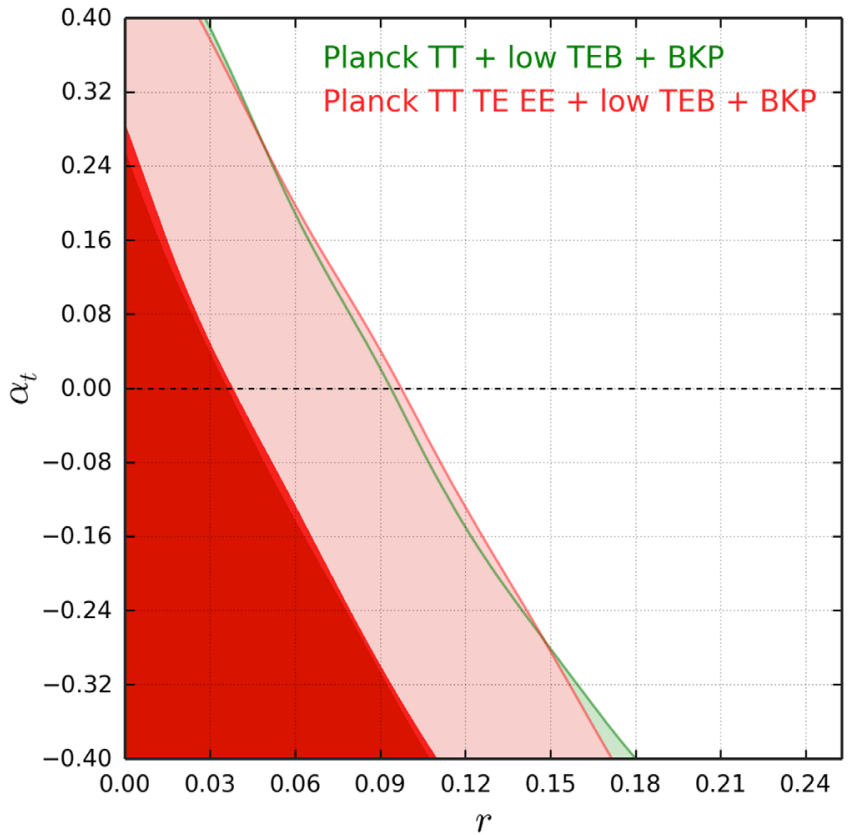

(95\% C.L., Planck TT TE EE + low TEB + BKP). In Figs. 3 and 4, we plot the marginalized contours at $68 \%$ and $95 \%$ C.L. in the planes $\left(r, \alpha_{\mathrm{t}}\right)$ and $\left(r, \beta_{\mathrm{t}}\right)$ both relaxing and imposing the consistency relations. Once again the first

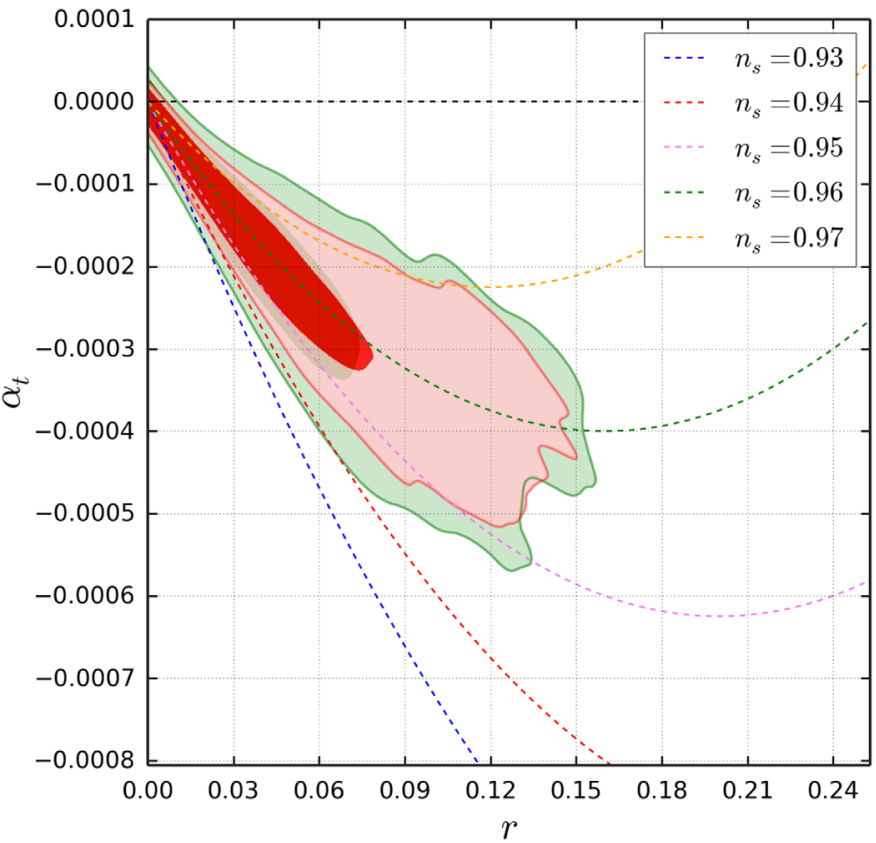

FIG. 3. Marginalized joint confidence contours at 68\% and 95\% C.L. in the plane $\left(\alpha_{\mathrm{t}}, r\right)$ for the indicated datasets. On the left (right) side panel the contours obtained relaxing (imposing) the consistency relations. The colored dashed lines represent the consistency relations (20) for different values of $n_{\mathrm{s}}$. 

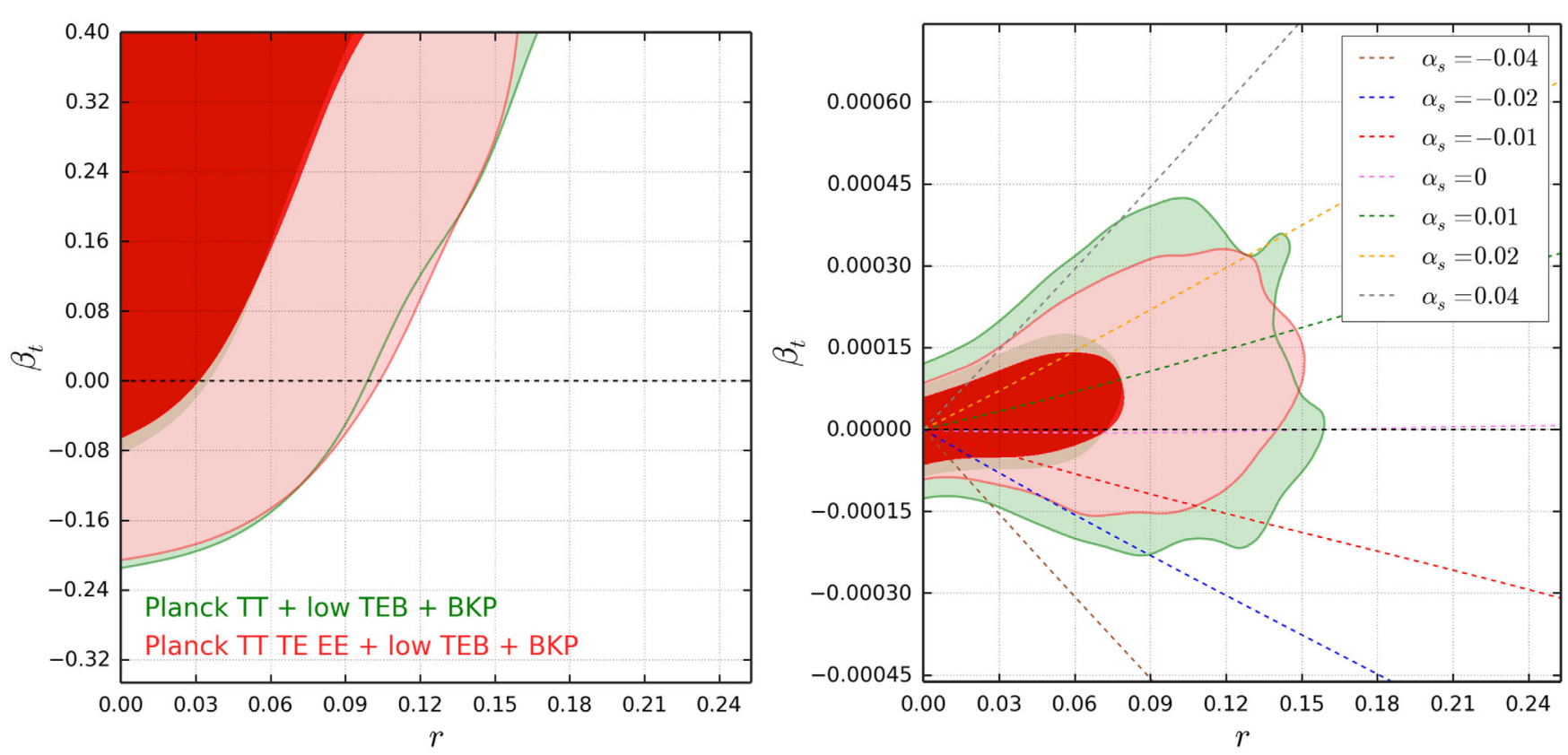

FIG. 4. Marginalized joint confidence contours at $68 \%$ and $95 \%$ C.L. in the plane $\left(\beta_{\mathrm{t}}, r\right)$ for the indicated datasets. On the left (right) side panel the contours obtained relaxing (imposing) the consistency relations. The colored dashed lines represent the consistency relations (21) for $n_{\mathrm{s}}=0.959$ and the different indicated values of $\alpha_{\mathrm{s}}$.

include the second. Together with the derived marginalized contours we also plot the curves that represent the consistency relations for different values of the scalar parameters (colored dashed lines in the right panels). From the right side panel of Fig. 3, one can see how imposing the consistency relations, regions with $\alpha_{\mathrm{t}}>0$, are almost excluded by data. On the other hand, from the right side panel of Fig. 4, we can see that both positive and negative values of $\beta_{\mathrm{t}}$ are possible.

Since the consistency relation (21) suggests that the sign of $\beta_{\mathrm{t}}$ may strongly depend on the value of $\alpha_{\mathrm{s}}$, it is interesting to study the situation in the plane $\left(\alpha_{\mathrm{s}}, \beta_{\mathrm{t}}\right)$.
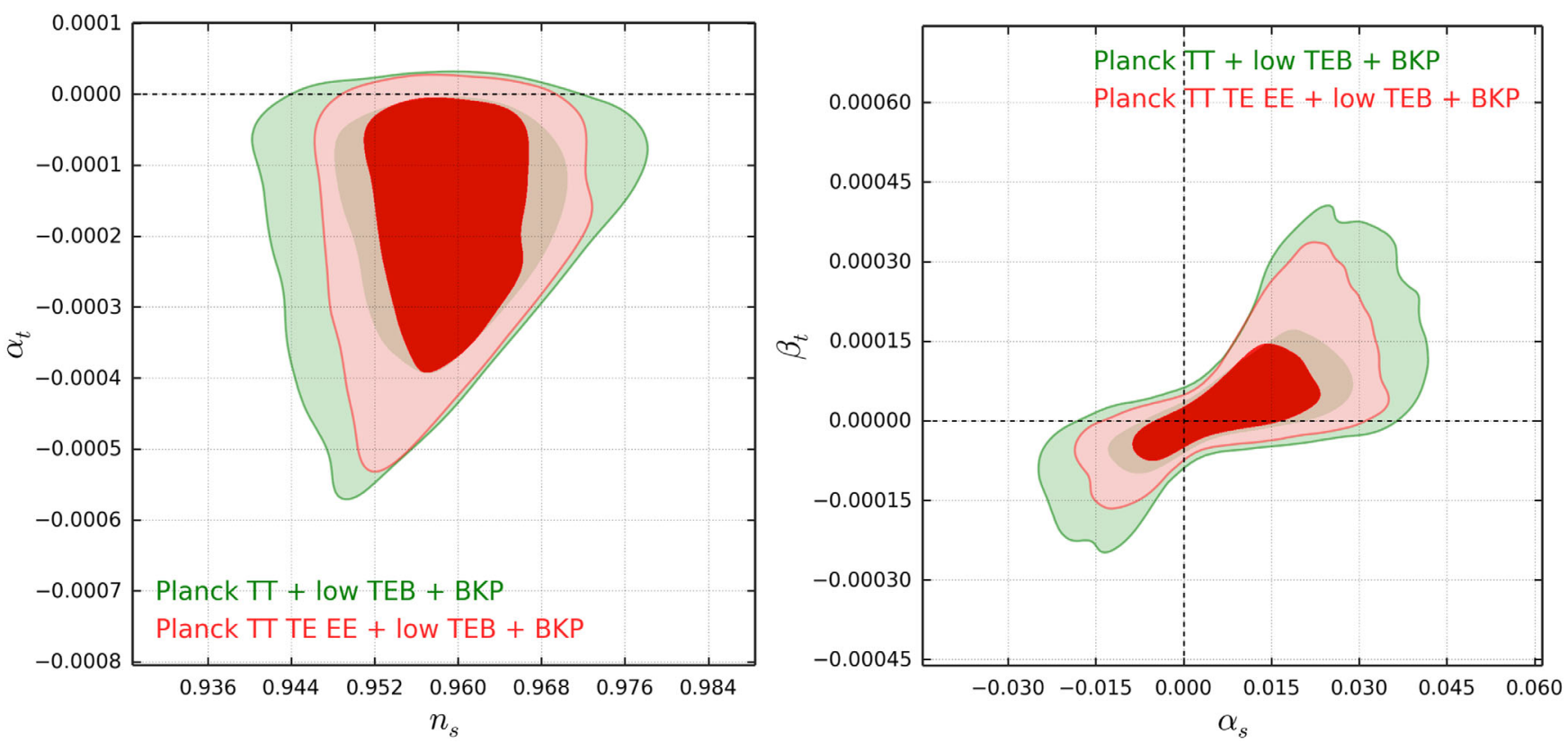

FIG. 5. Marginalized joint confidence contours at $68 \%$ and $95 \%$ C.L. obtained imposing the consistency relations for the indicated datasets. On the left side panels the plane $\left(n_{\mathrm{s}}, \alpha_{\mathrm{t}}\right)$. On the right side panel the plane $\left(\alpha_{\mathrm{s}}, \beta_{\mathrm{t}}\right)$. 

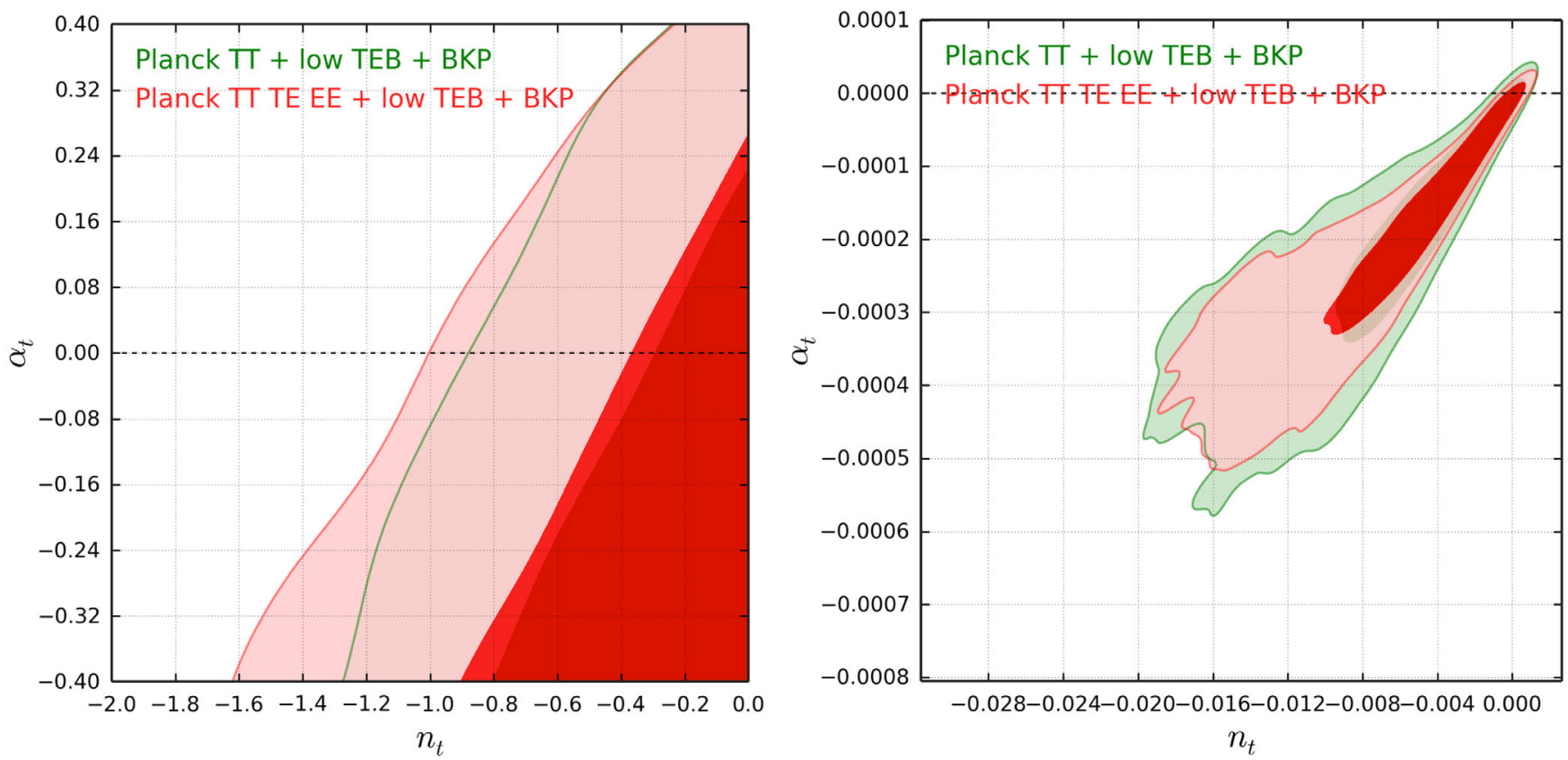

FIG. 6. Marginalized joint confidence contours at 68\% and 95\% C.L. in the plane $\left(n_{\mathrm{t}}, \alpha_{\mathrm{t}}\right)$ for the indicated datasets. On the left (right) panels the contours obtained relaxing (imposing) the consistency relations.

This is what we have done in the right side panel of Fig. 5 where we have plotted the marginalized contours at $68 \%$ and $95 \%$ C.L. in that plane, assuming the consistency relations. As one can see for negative values of $\alpha_{\mathrm{t}}$, negative values of $\beta_{\mathrm{t}}$ are preferred as well, even if it is possible to have negative (positive) $\alpha_{\mathrm{s}}$ and positive (negative) $\beta_{\mathrm{s}}$ around $\left|\alpha_{\mathrm{s}}\right| \sim 0$. In the left side panel of the same figure, instead, we have plotted the marginalized contours in the plane $\left(n_{\mathrm{s}}, \alpha_{\mathrm{t}}\right)$ that confirm the fact that regions with $\alpha_{\mathrm{t}}>0$, albeit noncompletely excluded, are strongly disadvantaged. Other interesting plots of the marginalized contours at $68 \%$ and $95 \%$ C.L, both for the free parameters and for the
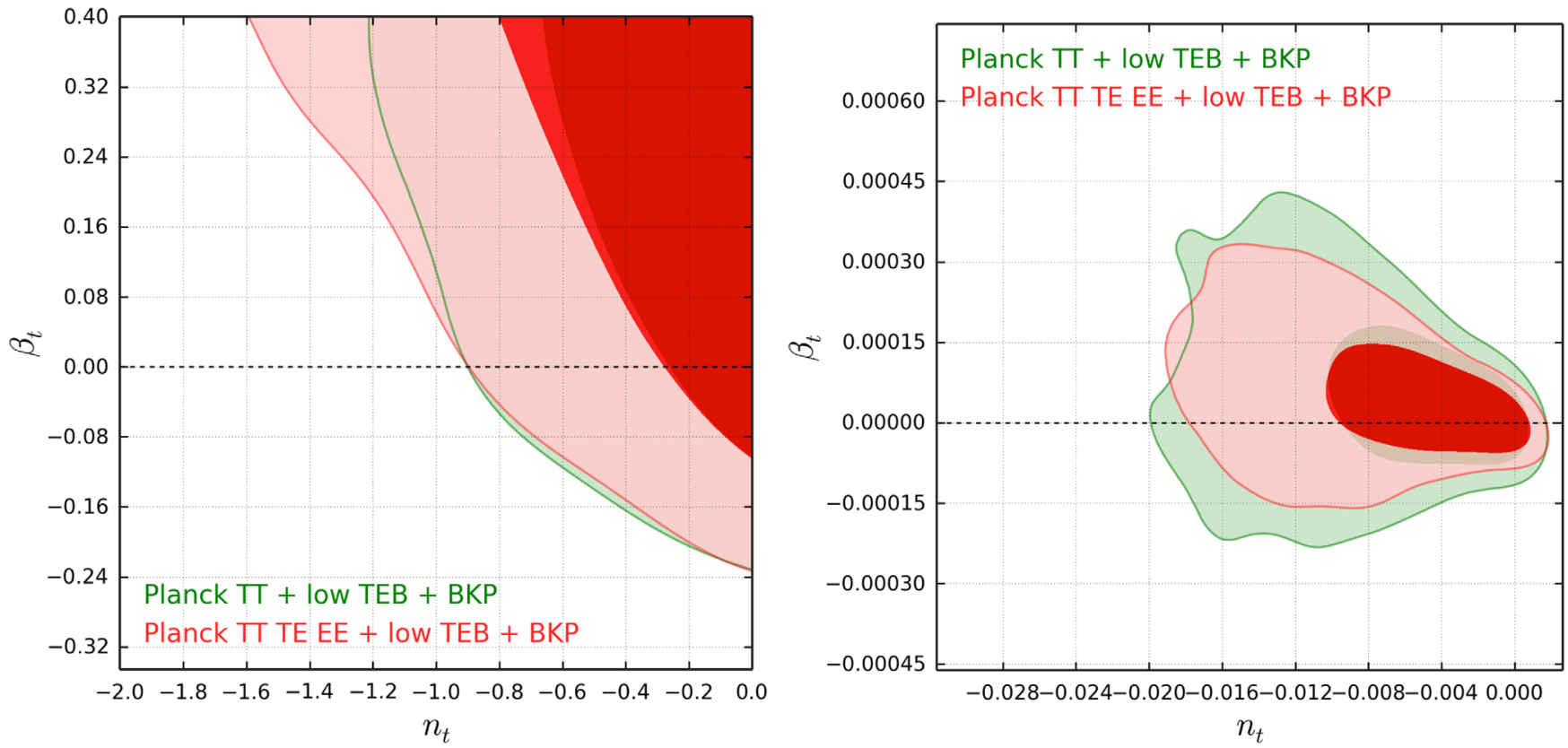

FIG. 7. Marginalized joint confidence contours at $68 \%$ and $95 \%$ C.L. in the plane $\left(n_{\mathrm{t}}, \beta_{\mathrm{t}}\right)$ for the indicated datasets. On the left (right) panels the contours obtained relaxing (imposing) the consistency relations. 

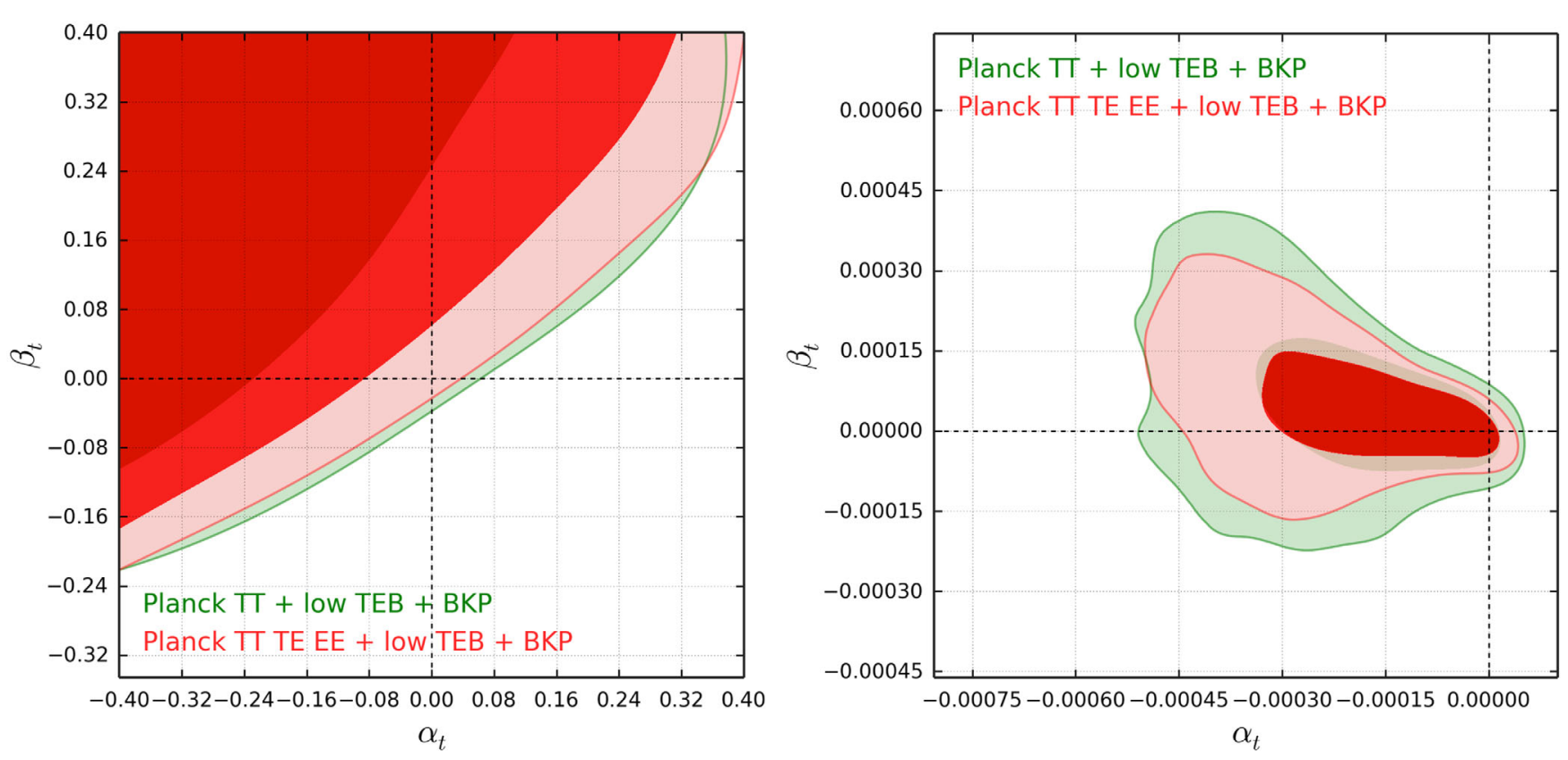

FIG. 8. Marginalized joint confidence contours at $68 \%$ and $95 \%$ C.L. in the plane $\left(\alpha_{\mathrm{t}}, \beta_{\mathrm{t}}\right)$ for the indicated datasets. On the left (right) panels the contours obtained relaxing (imposing) the consistency relations.

derived parameters, are given in Figs. 6-8 where in the left side panels are plotted the contours obtained relaxing the consistency relations while in the right side panels there are the respective contours obtained imposing them. Once again we can see how the second are fully embedded in the first. From the right side panels of Figs. 6 and 8, where the consistency relations are assumed, we can also see the previous considerations about a negative $\alpha_{\mathrm{t}}$ are still valid.

In Table IV we summarize all the 95\% C.L. limits for the tensor parameters, comparing the constraints obtained relaxing the consistency relations discussed in the previous subsection, with those obtained here fixing the single field slow-roll condition. The constraints obtained imposing the consistency relations are of course compatible with the larger bounds obtained leaving the parameters free.

\section{CONCLUSIONS}

In this paper we have first derived a set of consistency relations among the scalar and tensor inflationary parameters that are valid for the single field slow-roll inflationary models, proving also that, for such models, one can obtain as many relations as he wants. We focused on the consistency relations involving the tensor parameters $n_{\mathrm{t}}, \alpha_{\mathrm{t}}$, and $\beta_{\mathrm{t}}$ that can be expressed in terms of the tensor-to-scalar ratio $r$, the spectral index $n_{s}$, and the scalar running $\alpha_{\mathrm{s}}$ by Eqs. (19), (20), and (21). We also derived a fourth consistency relation for $\frac{d \beta_{\mathrm{t}}}{d \log k}$, Eq. (30). From these consistency relations, we argued that, for sufficiently small $r$, we would expect a negative tensor running $\alpha_{\mathrm{t}}$ while the sign of $\beta_{\mathrm{t}}$ depends on the sign and above all on the amplitude of the scalar running $\alpha_{\mathrm{s}}$. To check the slow-roll condition we performed a MCMC analysis considering an extended cosmological model with six additional parameters $\left(\alpha_{\mathrm{s}}, \beta_{\mathrm{s}}, r, n_{\mathrm{t}}, \alpha_{\mathrm{t}}\right.$, and $\beta_{\mathrm{t}}$ ) with respect to the standard $\Lambda \mathrm{CDM}$ model. We analyzed both the Planck CMB polarization and anisotropies 2015 data and the BKP data for the BB spectrum, normalizing the inflationary parameters at the pivot scale

TABLE IV. The 95\% C.L. bounds on the tensor parameters relaxing and imposing the consistency relations.

\begin{tabular}{lcccc}
\hline \hline Parameter & TT + low P & TT, TE, EE + low P & TT + low P + BKP & TT, TE, EE, +low P + BKP \\
\hline$r$ & $<0.785$ & $<0.629$ & $<0.127$ & $<0.124$ \\
$n_{\mathrm{t}}$ & $>-0.890$ & $>-0.907$ & $>-0.990$ & $>-1.15$ \\
& $>-0.120$ & $>-0.0800$ & $>-0.0158$ & $>-0.0157$ \\
$\alpha_{\mathrm{t}}$ & $<0.209$ & $<0.186$ & $<0.231$ & $<0.232$ \\
& $0.00096_{-0.0019}^{+0.0050}$ & $0.0003_{-0.0010}^{+0.0036}$ & $-0.00018_{-0.00025}^{+0.00019}$ & $-0.00018_{-0.00024}^{+0.00019}$ \\
$\beta_{\mathrm{t}}$ & $>-0.0707$ & $>-0.0667$ & $>-0.102$ & $>-0.0918$ \\
& $-0.0005_{-0.0045}^{+0.0011}$ & $-0.0001_{-0.0019}^{+0.0007}$ & $0.00004_{-0.00019}^{+0.00027}$ & $0.00004_{-0.00013}^{+0.00022}$ \\
\hline \hline
\end{tabular}


of $k_{*}=0.05 \mathrm{Mpc}^{-1}$. In Sec. IV we first presented the results obtained relaxing the consistency relations finding $r<0.127$ (95\% C.L., Planck TT + low P + BKP) and $r<0.124 \quad(95 \% \quad$ C.L., Planck TT TE EE + low $\mathrm{P}+\mathrm{BKP}$ ). For the tensor spectral index we found the lower bounds $n_{\mathrm{t}}>-0.990 \quad(95 \% \quad$ C.L., Planck TT + low P + BKP) and $n_{\mathrm{t}}>-1.15$ (95\% C.L., Planck TT TE EE + low $\mathrm{P}+\mathrm{BKP}$ ), while for the tensor running $\alpha_{\mathrm{t}}$ we found the upper limits $\alpha_{\mathrm{t}}<0.231$ (95\% C.L., Planck TT + low P + BKP) and $\alpha_{\mathrm{t}}<0.232$ (95\% C.L., Planck TT TE EE + low P + BKP). On the other hand, for the tensor running of running $\beta_{\mathrm{t}}$ we found the lower limits $\beta_{\mathrm{t}}>-0.102 \quad(95 \% \quad$ C.L., Planck $\mathrm{TT}+$ low $\mathrm{P}+\mathrm{BKP})$ and $\beta_{\mathrm{t}}>-0.0918 \quad(95 \% \quad$ C.L., Planck TT TE EE + low P + BKP).

Then we have fixed the consistency relations among the inflationary parameters, and we have also presented the constraints on the derived parameters. We have compared these new constraints with the previous ones in order to check the slow-roll condition. For the derived tensor spectral index $n_{\mathrm{t}}$ we found $n_{\mathrm{t}}>-0.0158$ (95\% C.L., Planck TT + low TEB + BKP) and $n_{\mathrm{t}}>-0.0157$ (95\% C.L., Planck TT TE EE + low TEB + BKP) while for the derived tensor runnings $\alpha_{\mathrm{t}}$ and $\beta_{\mathrm{t}}$ we found, respectively, $\alpha_{\mathrm{t}}=-0.00018_{-0.00025}^{+0.0019} \quad(95 \%$ C.L., Planck TT + low TEB + BKP), $\alpha_{\mathrm{t}}=-0.00018_{-0.00024}^{+0.00019}(95 \%$ C.L., Planck TT TE EE + low TEB + BKP), and $\beta_{\mathrm{t}}=$ $0.00004_{-0.00019}^{+0.00027} \quad(95 \% \quad$ C.L., $\quad$ Planck TT + low TEB + BKP), $\beta_{\mathrm{t}}=0.00004_{-0.00013}^{+0.00022}(95 \%$ C.L., Planck TT TE $\mathrm{EE}+$ low TEB + BKP). Therefore the 95\% C.L. constraints obtained from the consistency relations, in addition to being much tighter, are also in accordance with the larger limits obtained relaxing the consistency relations.

\section{ACKNOWLEDGMENTS}

E. D. V. acknowledges support from the European Research Council in the form of Consolidator Grant No. 681431. A. M. thanks the University of Manchester and the Jodrell Bank Center for Astrophysics for hospitality. A. M. and W. G. are supported by TASP, iniziativa specifica INFN. We thank Nicola Bartolo for useful discussions.

\section{APPENDIX: BEYOND THE LEADING ORDER IN THE PRIMORDIAL SPECTRA}

In Sec. II we have explicitly derived some consistency relations [Eqs. (19)-(21) and Eq. (30)], and we have also shown that consistency relations can be found at any order. However, all the consistency relations that we have derived come from the primordial spectra at leading order in the slow-roll approximation. So one could ask if considering the primordial spectra at the leading order is a good approximation for such high order consistency relations and, in general, what happens if one goes beyond the leading order. What we want to show is that if one goes beyond the leading order, each equation is corrected by higher order terms that, within the same equation, are negligible.

Let us start our discussion going beyond the leading order spectra. At the next order in the slow-roll approximations they read $[8,9]$

$$
\begin{array}{r}
\mathcal{P}_{\mathrm{s}}=\frac{H^{2}}{8 \pi^{2} M_{\mathrm{P}}^{2} \epsilon_{1}}\left\{1-2(C+1) \epsilon_{1}-C \epsilon_{2}\right\}+\mathcal{O}\left(\epsilon^{2}\right), \\
\mathcal{P}_{\mathrm{t}}=\frac{2 H^{2}}{\pi^{2} M_{\mathrm{P}}^{2}}\left\{1-2(1+C) \epsilon_{1}\right\}+\mathcal{O}\left(\epsilon^{2}\right),
\end{array}
$$

where $C \equiv \gamma+\ln 2-2 \approx-0.73$. Since the logarithmic derivative of a quantity of order $\epsilon^{n}$ is of order $\epsilon^{n+1}$, we immediately see that all the corrections of order $\epsilon$ in the primordial spectra become corrections of order $\epsilon^{2}$ in the scalar and tensor running $n_{\mathrm{s}, \mathrm{t}}, \epsilon^{3}$ in their running $\alpha_{\mathrm{s}, \mathrm{t}}, \epsilon^{4}$ in their running of running $\beta_{\mathrm{s}, \mathrm{t}}$, and so on. In fact, a straightforward computation gives

$$
\begin{gathered}
n_{\mathrm{s}}-1=-2 \epsilon_{1}-\epsilon_{2}+\underbrace{\left[-2 \epsilon_{1}^{2}-(3+2 C) \epsilon_{1} \epsilon_{2}-C \epsilon_{2} \epsilon_{3}\right]}_{\mathcal{O}\left(\epsilon^{2}\right)}, \\
n_{\mathrm{t}}=-2 \epsilon_{1}+\underbrace{\left[-2 \epsilon_{1}^{2}-2(1+C) \epsilon_{1} \epsilon_{2}\right]}_{\mathcal{O}\left(\epsilon^{2}\right)} .
\end{gathered}
$$

Therefore the systems (25) and (26) remain the same except for higher order corrections:

$$
\begin{aligned}
n_{\mathrm{s}}-1 & =-2 \epsilon_{1}-\epsilon_{2}+\mathcal{O}\left(\epsilon^{2}\right), \\
\frac{d n_{\mathrm{s}}}{d \log k} & \equiv \alpha_{\mathrm{s}}=-2 \epsilon_{1} \epsilon_{2}-\epsilon_{2} \epsilon_{3}+\mathcal{O}\left(\epsilon^{3}\right), \\
\frac{d^{2} n_{\mathrm{s}}}{d(\log k)^{2}} & \equiv \beta_{\mathrm{s}}=-2 \epsilon_{1} \epsilon_{2}^{2}-2 \epsilon_{1} \epsilon_{2} \epsilon_{3}-\epsilon_{2} \epsilon_{3}^{2}-\epsilon_{2} \epsilon_{3} \epsilon_{4}+\mathcal{O}\left(\epsilon^{4}\right), \\
\ldots & \\
\frac{d^{n-1} n_{\mathrm{s}}}{d(\log k)^{n-1}} & =f_{n}\left(\epsilon_{1}, \ldots, \epsilon_{n+1}\right)+\mathcal{O}\left(\epsilon^{n+1}\right),
\end{aligned}
$$


and

$$
\begin{aligned}
n_{\mathrm{t}} & =-2 \epsilon_{1}+\mathcal{O}\left(\epsilon^{2}\right), \\
\frac{d n_{\mathrm{t}}}{d \log k} & \equiv \alpha_{\mathrm{t}}=-2 \epsilon_{1} \epsilon_{2}+\mathcal{O}\left(\epsilon^{3}\right), \\
\frac{d^{2} n_{\mathrm{t}}}{d(\log k)^{2}} & \equiv \beta_{\mathrm{t}}=-2 \epsilon_{1} \epsilon_{2}^{2}-2 \epsilon_{1} \epsilon_{2} \epsilon_{3}+\mathcal{O}\left(\epsilon^{4}\right), \\
\frac{d^{3} n_{\mathrm{t}}}{d(\log k)^{3}} & =\frac{d \beta_{\mathrm{t}}}{d \log (k)}=-2 \epsilon_{1} \epsilon_{2}^{3}-6 \epsilon_{1} \epsilon_{2}^{2} \epsilon_{3}-2 \epsilon_{1} \epsilon_{2} \epsilon_{3}^{2}-2 \epsilon_{1} \epsilon_{2} \epsilon_{3} \epsilon_{4}+\mathcal{O}\left(\epsilon^{5}\right), \\
& \ldots \\
\frac{d^{n} n_{\mathrm{t}}}{d(\log k)^{n}} & =g_{n+1}\left(\epsilon_{1}, \ldots, \epsilon_{n+1}\right)+\mathcal{O}\left(\epsilon^{n+2}\right) .
\end{aligned}
$$

So following the derivation given in Sec. II, one can see that all the possible corrections to our consistency relations are negligible,

$$
\begin{aligned}
r & =-8 n_{\mathrm{t}}+\mathcal{O}\left(\epsilon^{2}\right), \\
n_{\mathrm{s}}-1 & =n_{\mathrm{t}}-\frac{\alpha_{\mathrm{t}}}{n_{\mathrm{t}}}+\mathcal{O}\left(\epsilon^{2}\right), \\
\frac{d n_{\mathrm{s}}}{d \log k} & \equiv \alpha_{\mathrm{s}}=\alpha_{\mathrm{t}}+\left(\frac{\alpha_{\mathrm{t}}}{n_{\mathrm{t}}}\right)^{2}-\frac{\beta_{\mathrm{t}}}{n_{\mathrm{t}}}+\mathcal{O}\left(\epsilon^{3}\right), \\
\frac{d^{2} n_{\mathrm{s}}}{d(\log k)^{2}} & \equiv \beta_{\mathrm{s}}=\beta_{\mathrm{t}}-2\left(\frac{\alpha_{\mathrm{t}}^{3}}{n_{\mathrm{t}}^{3}}\right)+3\left(\frac{\alpha_{\mathrm{t}} \beta_{\mathrm{t}}}{n_{\mathrm{t}}^{2}}\right)-\frac{1}{n_{\mathrm{t}}}\left(\frac{d^{3} n_{\mathrm{t}}}{d(\log k)^{3}}\right)+\mathcal{O}\left(\epsilon^{4}\right), \\
\ldots & \\
\frac{d^{n-1} n_{\mathrm{s}}}{d(\log k)^{n-1}} & =\tilde{f}_{n}\left(n_{\mathrm{t}}, \alpha_{\mathrm{t}}, \beta_{\mathrm{t}}, \frac{d^{3} n_{\mathrm{t}}}{d(\log k)^{3}}, \ldots, \frac{d^{n} n_{\mathrm{t}}}{d(\log k)^{n}}\right)+\mathcal{O}\left(\epsilon^{n+1}\right),
\end{aligned}
$$

and so
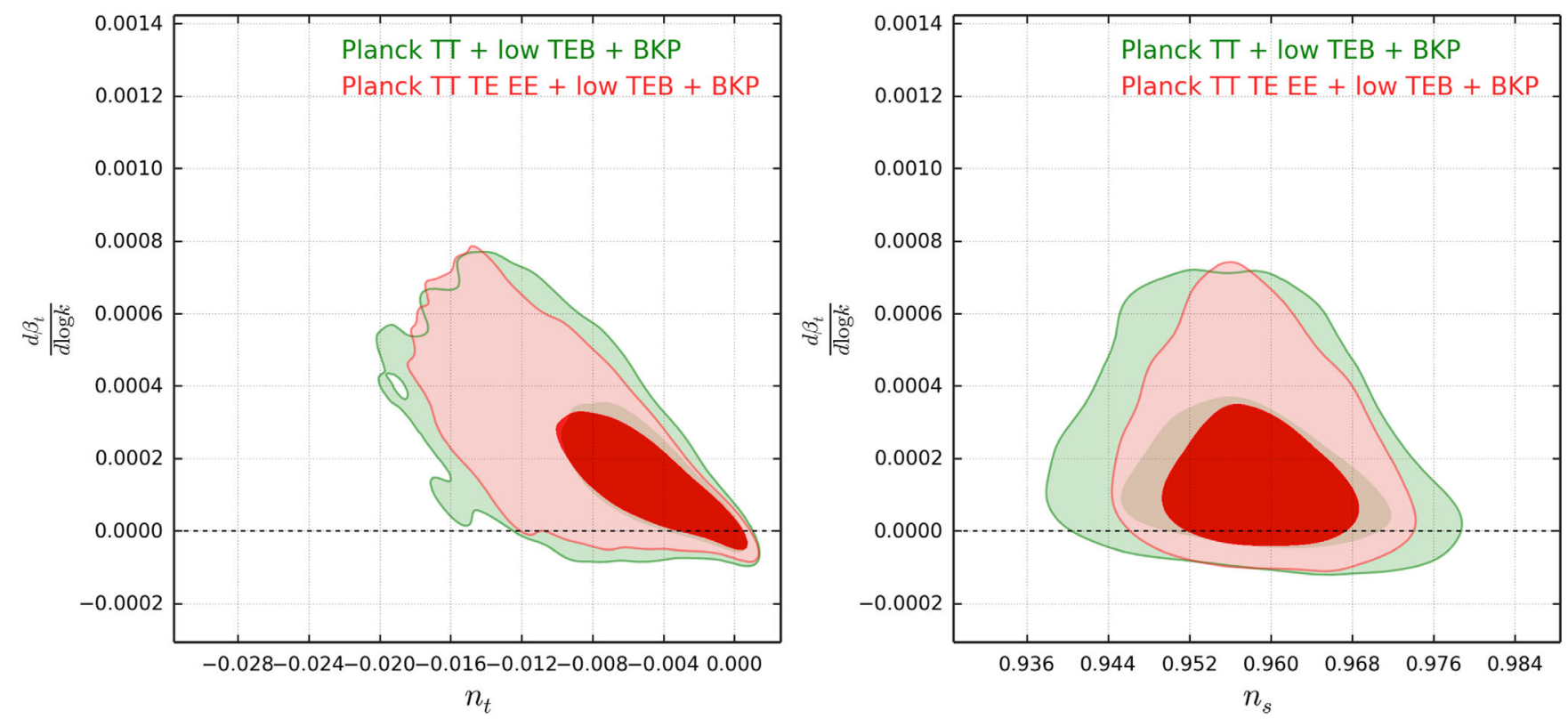

FIG. 9. Marginalized joint confidence contours at 68\% and 95\% C.L. in the planes $\left(n_{\mathrm{t}}, \frac{d \beta_{\mathrm{t}}}{d \log k}\right)$ (left panels) and $\left(n_{\mathrm{s}}, \frac{d \beta_{\mathrm{t}}}{d \log k}\right)$ (right panels), for the indicated datasets. 

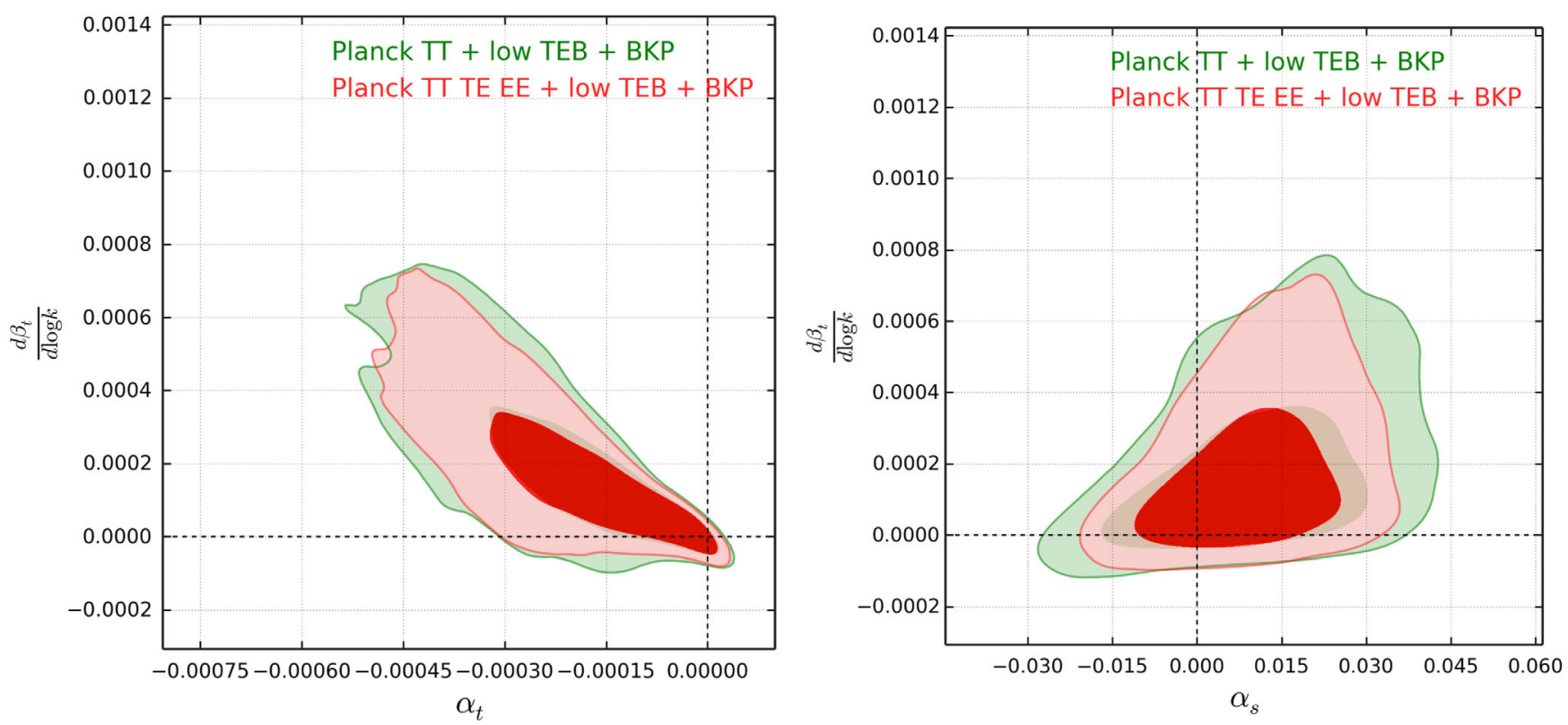

FIG. 10. Marginalized joint confidence contours at $68 \%$ and 95\% C.L. in the planes $\left(\alpha_{\mathrm{t}}, \frac{d \beta_{\mathrm{t}}}{d \log k}\right)$ (left panels) and $\left(\alpha_{\mathrm{s}}, \frac{d \beta_{1}}{d \log k}\right)($ right panels), for the indicated datasets.

$$
\begin{gathered}
n_{\mathrm{t}}=\underbrace{-\frac{r}{8}}_{\mathcal{O}(\epsilon)}+\mathcal{O}\left(\epsilon^{2}\right), \\
\alpha_{\mathrm{t}}=\underbrace{\frac{r}{8}\left(n_{s}-1\right)+\frac{r^{2}}{64}}_{\mathcal{O}\left(\epsilon^{2}\right)}+\mathcal{O}\left(\epsilon^{3}\right),
\end{gathered}
$$

$$
\beta_{\mathrm{t}}=\underbrace{\frac{r}{8}\left[\alpha_{\mathrm{s}}-\left(n_{\mathrm{s}}-1\right)^{2}\right]-\frac{3 r^{2}}{64}\left(n_{\mathrm{s}}-1\right)-\frac{r^{3}}{256}}_{\mathcal{O}\left(\epsilon^{3}\right)}+\mathcal{O}\left(\epsilon^{4}\right) .
$$

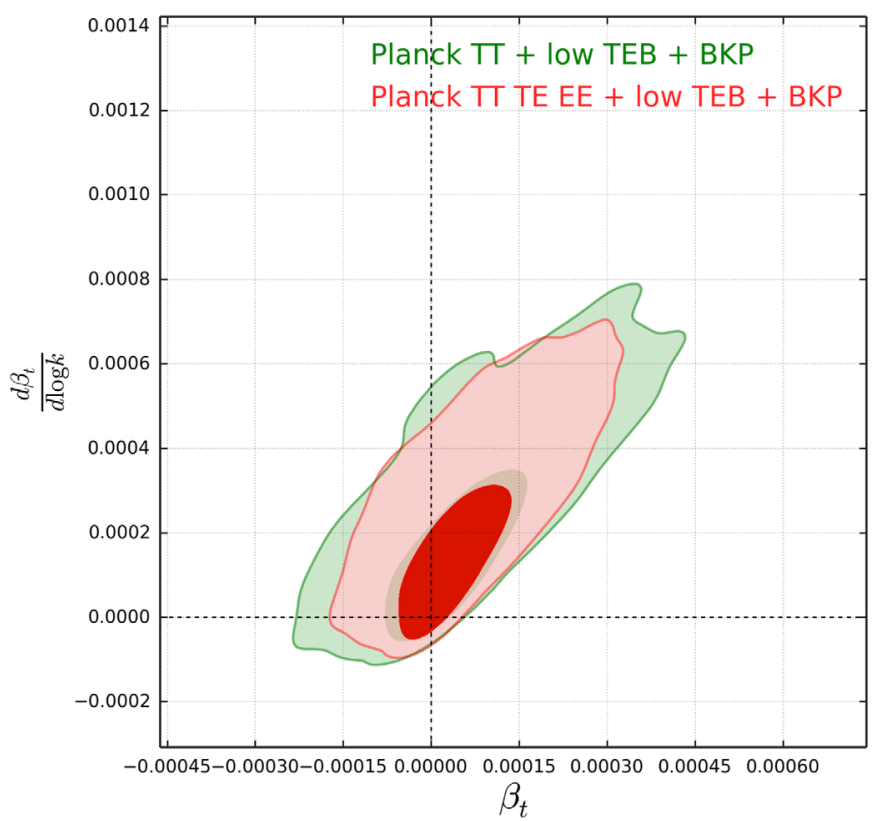

FIG. 11. Marginalized joint confidence contours at $68 \%$ and $95 \%$ C.L. in the planes $\left(\beta_{\mathrm{t}}, \frac{d \beta_{\mathrm{t}}}{d \log k}\right)$ (left panels) and $\left(\beta_{\mathrm{s}}, \frac{d \beta_{\mathrm{t}}}{d \log k}\right)($ right panels), for the indicated datasets.

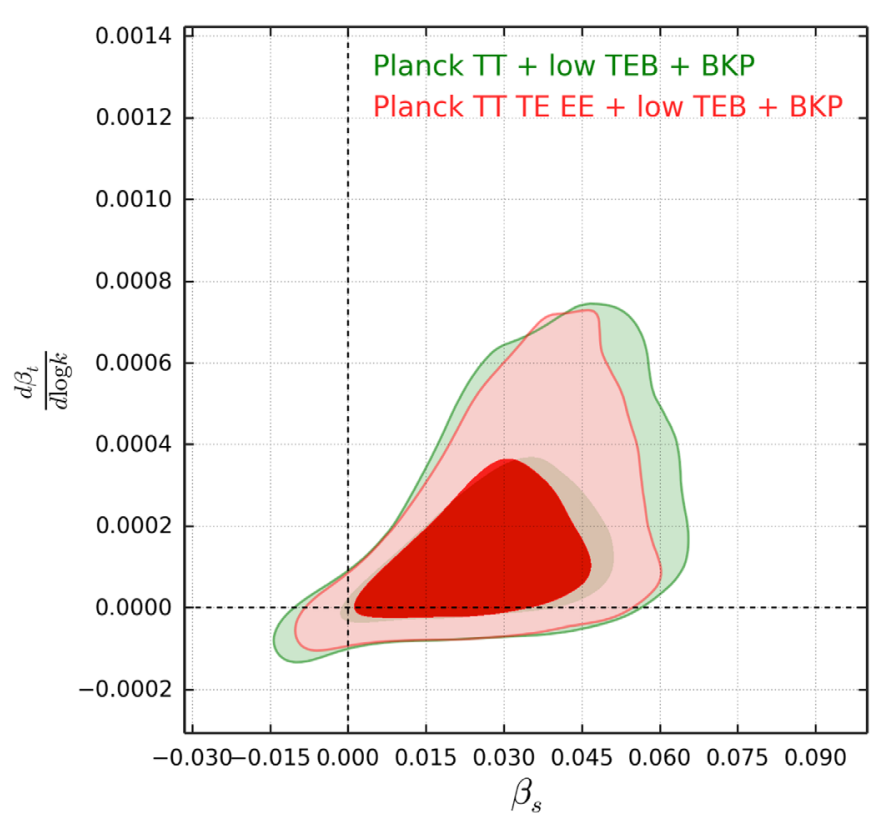

For example, one can compute the correction to the consistency relation $n_{\mathrm{t}}=-\frac{r}{8}$ to obtain $[8,9,48,50]$ 


$$
n_{\mathrm{t}}=-\frac{r}{8}-\frac{r}{8}\left[-\frac{r}{16}+1-n_{S}\right]+\mathcal{O}\left(\epsilon^{3}\right)
$$

where the correction of order $\mathcal{O}\left(\epsilon^{3}\right)$ has to be computed including corrections of order $\epsilon^{2}$ in the primordial spectra. One can immediately see that the correction $\Delta n_{\mathrm{t}}=$ $-\frac{r}{8}\left[-\frac{r}{16}+1-n_{S}\right]$ is less than the $3 \%$ for $n_{\mathrm{s}} \approx 0.96$ and the maximum value of $r_{\max }=0.24$ used in our analysis in Sec. IV.

Clearly, generalizing this discussion, further corrections of order $\epsilon^{n}$ in the spectra will provide corrections of order $\epsilon^{n+1}$ in the scalar and tensor tilts, $\epsilon^{n+2}$ in their running, $\epsilon^{n+3}$ in their running of running, and so on. All these contributions are of course smaller and smaller.

\section{Constraints on $\gamma_{\mathrm{t}}=\frac{d \beta_{\mathrm{t}}}{d \log k}$}

As stated in Sec. II, since in our simulations we have parametrized the scalar spectrum including the running of running $\beta_{\mathrm{s}}$, thanks to Eqs. (19), (20), (21), and (30), we can also provide the constraints on $\gamma_{\mathrm{t}}=\frac{d \beta_{\mathrm{t}}}{d \log k}$ that, for the different datasets, are presented in Table $\mathrm{V}$ at $95 \%$ C.L.
TABLE V. $\quad 95 \%$ C.L. bounds on $\gamma_{\mathrm{t}}=\frac{d \beta_{\mathrm{t}}}{d \log k}$ from the consistency relations for different datasets.

\begin{tabular}{lc}
\hline \hline Dataset & $\gamma_{\mathrm{t}}=\frac{d \beta_{\mathrm{t}}}{d \log k} 95 \%$ C.L. \\
\hline Planck TT + low P & $0.0009_{-0.0012}^{+0.0024}$ \\
Planck TT TE EE + low P & $0.00068_{-0.000080}^{+0.0019}$ \\
Planck TT + low P + BKP & $0.00017_{-0.00044}^{+0.00021}$ \\
Planck TT TE EE + low P + BKP & $0.00017_{-0.00040}^{+0.00019}$ \\
\hline \hline
\end{tabular}

In Figs. 9-11 we also plot the derived marginalized contours at $68 \%$ and $95 \%$ C.L. in different planes for $\gamma_{t}$ obtained imposing the consistency relations. In the left panels we present the contours among $\gamma_{\mathrm{t}}$ and the other tensor parameters while in the right panels we focus on the contours for $\gamma_{\mathrm{t}}$ and the scalar parameters. As well as for $\beta_{\mathrm{t}}$ also in this case, positive values for $\gamma_{\mathrm{t}}$ seems to be favored by data. We remark that the constraints for $\gamma_{\mathrm{t}}=\frac{d \beta_{\mathrm{t}}}{d \log k}$ completely derive from the consistency relations since in our simulations we have not included this effect in the spectral parametrization.
[1] D. H. Lyth, Phys. Rev. Lett. 78, 1861 (1997).

[2] D. Baumann, D. Green, and R. A. Porto, J. Cosmol. Astropart. Phys. 01 (2015) 016.

[3] D. H. Lyth, J. Cosmol. Astropart. Phys. 08 (2013) 007.

[4] A. R. Liddle and S. M. Leach, Phys. Rev. D 68, 103503 (2003).

[5] M. Kamionkowski and E. D. Kovetz, Annu. Rev. Astron. Astrophys. 54, 227 (2016).

[6] R. R. Caldwell, T. L. Smith, and D. G. E. Walker, arXiv:1812.07577.

[7] Y. Akrami, J. Socorro, and R. Hernández-Jiménez (Planck Collaboration), Astrophys. Space Sci. 364, 69 (2019).

[8] A. R. Liddle and M. S. Turner, Phys. Rev. D 50, 758 (1994); 54, 2980(E) (1996).

[9] J. E. Lidsey, A. R. Liddle, E. W. Kolb, E. J. Copeland, T. Barreiro, and M. Abney, Rev. Mod. Phys. 69, 373 (1997).

[10] T. Damour and V. F. Mukhanov, Phys. Rev. Lett. 80, 3440 (1998).

[11] A. H. Guth, Phys. Rev. D 23, 347 (1981); Adv. Ser. Astrophys. Cosmol. 3, 139 (1987).

[12] A. H. Guth, D. I. Kaiser, and Y. Nomura, Phys. Lett. B 733, 112 (2014).

[13] A. D. Linde, Phys. Lett. 108B, 389 (1982); Adv. Ser. Astrophys. Cosmol. 3, 149 (1987).

[14] A. Vilenkin, Phys. Rev. D 27, 2848 (1983).

[15] A. S. Goncharov, A. D. Linde, and V. F. Mukhanov, Int. J. Mod. Phys. A 02, 561 (1987).

[16] D. Baumann, Inflation https://doi.org/10.1142/ 9789814327183_0010 [arXiv:0907.5424].

[17] S. W. Hawking, Phys. Lett. 115B, 295 (1982).
[18] A. Albrecht and P. J. Steinhardt, Phys. Rev. Lett. 48, 1220 (1982); Adv. Ser. Astrophys. Cosmol. 3, 158 (1987).

[19] A. A. Starobinsky, Phys. Lett. 91B, 99 (1980); 91B, 99 (1980); Adv. Ser. Astrophys. Cosmol. 3, 130 (1987).

[20] A. D. Linde, Phys. Lett. 129B, 177 (1983).

[21] A. D. Linde, Phys. Rev. D 49, 748 (1994).

[22] C. Armendariz-Picon, T. Damour, and V. F. Mukhanov, Phys. Lett. B 458, 209 (1999).

[23] E. J. Copeland, A. R. Liddle, D. H. Lyth, E. D. Stewart, and D. Wands, Phys. Rev. D 49, 6410 (1994).

[24] A. D. Linde, Phys. Lett. B 175, 395 (1986).

[25] D. H. Lyth and A.R. Liddle, The Primordial Density Perturbation: Cosmology, Inflation and the Origin of Structure (Cambridge University Press, Cambridge, England, 2009), p. 497.

[26] V. Mukhanov, Physical Foundations of Cosmology.

[27] S. Dodelson, Modern Cosmology (Academic Press, Amsterdam, 2003), p. 440.

[28] S. Weinberg, Cosmology (Oxford University Press, Oxford, 2008), p. 593.

[29] A. H. Guth and S. Y. Pi, Phys. Rev. D 32, 1899 (1985).

[30] A. A. Starobinsky, Pis'ma Zh. Eksp. Teor. Fiz. 55, 477 (1992) [JETP Lett. 55, 489 (1992)].

[31] A. A. Starobinsky, Pis'ma Zh. Eksp. Teor. Fiz. 30, 719 (1979) [JETP Lett. 30, 682 (1979)].

[32] A. A. Starobinsky, Sov. Astron. Lett. 9, 302 (1983).

[33] V. F. Mukhanov and G. V. Chibisov, Pis'ma Zh. Eksp. Teor. Fiz. 33, 549 (1981) [JETP Lett. 33, 532 (1981)].

[34] V. F. Mukhanov, H. A. Feldman, and R. H. Brandenberger, Phys. Rep. 215, 203 (1992). 
[35] V. Mukhanov, Eur. Phys. J. C 73, 2486 (2013).

[36] V. F. Mukhanov and G. V. Chibisov, Zh. Eksp. Teor. Fiz. 83, 475 (1982) [Sov. Phys. JETP 56, 258 (1982)].

[37] J. M. Bardeen, Phys. Rev. D 22, 1882 (1980).

[38] J. M. Bardeen, P. J. Steinhardt, and M. S. Turner, Phys. Rev. D 28, 679 (1983).

[39] F. C. Adams, J. R. Bond, K. Freese, J. A. Frieman, and A. V. Olinto, Phys. Rev. D 47, 426 (1993).

[40] N. Bartolo, S. Matarrese, and A. Riotto, Phys. Rev. D 64, 123504 (2001).

[41] J. Choe, J. O. Gong, and E. D. Stewart, J. Cosmol. Astropart. Phys. 07 (2004) 012.

[42] C. Gordon, D. Wands, B. A. Bassett, and R. Maartens, Phys. Rev. D 63, 023506 (2000).

[43] M. G. Jackson and G. Shiu, Phys. Rev. D 88, 123511 (2013).

[44] J. Martin and D. J. Schwarz, Phys. Rev. D 67, 083512 (2003).

[45] J. A. Adams, B. Cresswell, and R. Easther, Phys. Rev. D 64, 123514 (2001).

[46] D. H. Lyth and D. Wands, Phys. Lett. B 524, 5 (2002).

[47] P. A. R. Ade et al. (Planck Collaboration), Astron. Astrophys. 571, A22 (2014).

[48] A. Kosowsky and M. S. Turner, Phys. Rev. D 52, R1739 (1995).
[49] J. Martin, C. Ringeval, and V. Vennin, Phys. Dark Universe 5-6, 75 (2014).

[50] A. R. Liddle and D. H. Lyth, Phys. Rep. 231, 1 (1993).

[51] R. L. Davis, H. M. Hodges, G. F. Smoot, P. J. Steinhardt, and M. S. Turner, Phys. Rev. Lett. 69, 1856 (1992); 70, 1733(E) (1993).

[52] D. S. Salopek, Phys. Rev. Lett. 69, 3602 (1992).

[53] A. R. Liddle and D. H. Lyth, Phys. Lett. B 291, 391 (1992).

[54] T. Souradeep and V. Sahni, Mod. Phys. Lett. A 07, 3541 (1992).

[55] A. Lewis and S. Bridle, Phys. Rev. D 66, 103511 (2002).

[56] N. Aghanim et al. (Planck Collaboration), Astron. Astrophys. 594, A11 (2016).

[57] A. Lewis, Phys. Rev. D 87, 103529 (2013).

[58] P. A. R. Ade et al. (Planck Collaboration), Astron. Astrophys. 594, A13 (2016).

[59] P. A. R. Ade et al. (BICEP2 and Planck Collaborations), Phys. Rev. Lett. 114, 101301 (2015).

[60] P. A. R. Ade et al. (Planck Collaboration), Astron. Astrophys. 594, A20 (2016).

[61] G. Cabass, E. Di Valentino, A. Melchiorri, E. Pajer, and J. Silk, Phys. Rev. D 94, 023523 (2016).

[62] G. Cabass, L. Pagano, L. Salvati, M. Gerbino, E. Giusarma, and A. Melchiorri, Phys. Rev. D 93, 063508 (2016). 